\title{
共价有机框架的合成及其在肿瘤治疗中的应用研究进展
}

\author{
王涛赵璐王科伟白云峰* 冯锋* \\ (山西大同大学化学与化工学院 化学生物传感山西省重点实验室 大同 037009)
}

\begin{abstract}
摘要 共价有机框架(covalent organic frameworks, COFs)是近年来开发的一种由有机单元连接而成的高结晶性多孔聚 合物, 由于具有良好的孔隙率、模块性、结晶性和生物相容性等特点在肿瘤治疗中显示出了良好的应用前景. 本综述 总结了已报道的 COFs 制备方法, 包括溶剂热合成法、机械化学合成法、微波合成法、离子热合成法、界面合成法、 室温合成法和纳米尺度 COFs 的合成方法, 并根据对肿瘤作用机理的差异, 将用于肿瘤治疗的 COFs 纳米载药系统归纳 为药物化疗、光热治疗、光动力学治疗和联合治疗. 此外还讨论了 $\mathrm{COFs}$ 在肿瘤治疗领域所面临的主要挑战和发展趋 势.
\end{abstract}

关键词 共价有机框架; 纳米材料; 肿瘤治疗; 药物载体

\section{Research Progress on the Synthesis of Covalent Organic Frameworks and Their Applications in Tumor Therapy}

\author{
Tao Wang Lu Zhao Kewei Wang Yunfeng Bai* Feng Feng* \\ (College of Chemistry and Chemical Engineering, Shanxi Provincial Key Laboratory of Chemical Biosensing, \\ Shanxi Datong University, Datong 037009, China)
}

\begin{abstract}
Covalent organic frameworks (COFs) are a class of crystalline porous polymer composed of organic units developed in recent years. Due to their good porosity, modularity, crystallinity and biocompatibility, they show a good application prospect in tumor therapy. The porous channels with adjustable pore sizes of COFs make them become an ideal drug delivery material. In addition, COFs can also be used in photothermal therapy and photodynamic therapy in combination with photothermal agents and photosensitizers. This review systematically introduces the synthesis methods of COFs, including solvothermal synthesis, mechanochemical synthesis, microwave synthesis, ionothermal synthesis, interfacial synthesis, room temperature synthesis and nanoscale synthesis. According to the differences in the mechanism of tumor therapy, the applications of COFs nanocarrier system for tumor therapy were reviewed, including chemotherapy, photothermal therapy, photodynamic therapy and combined therapy, proving that the promising drug carrier can effectively improve the therapeutic effect of nanocarriers on tumors. In addition, the main challenges and development trends of the COFs in the field of tumor therapy are discussed. This review could inspire research to design more effective COFs nano-drug delivery systems and promote the development of tumor therapy.
\end{abstract}

Keywords covalent organic framework; nanomaterial; tumor therapy; drug carrier

\section{1 引言}

目前, 人们对癌症的发病机理已经有了较为深入的 了解, 但是癌症的致死率仍然居高不下, 2018 年, 美国 共有 2813503 例死亡, 其中 $21 \%$ 是由癌症造成 ${ }^{[1]}$. 癌症 的诊疗分为早期诊断和后期治疗, 癌症的早期诊断对于 提高癌症患者的生存率具有极为重要的意义, 早期诊断 方法包括 CT、磁共振、肿瘤标志物检测等 ${ }^{[2-7]}$; 癌症的 后期治疗方法包括手术、放疗、化疗、免疫治疗和它们 之间的联合疗法等. 其中, 化疗是最为主流的治疗方法, 这种疗法对癌细胞毒性强、见效快、可以直接杀死癌细
胞 ${ }^{[8]}$, 但是它的不足是缺乏对癌细胞的靶向性, 会对人 体产生较强的毒副作用, 并且化疗药物的稳定性和新陈 代谢能力较差, 这些都极大地影响了化学疗法在抗击癌 症方面的应用，而纳米载药技术的应用可以改善药物化 疗的缺陷 ${ }^{[9]}$. 纳米载药系统由于具有对实体瘤的高通透 性和滞留效应(EPR 效应)增强了载药系统对肿瘤部位靶 向传递的能力, 使得它们在肿瘤部位的浓度极大地提 高 ${ }^{[10]}$. 纳米药物载体目前包括脂质体 ${ }^{[11]}$ 、碳纳米管

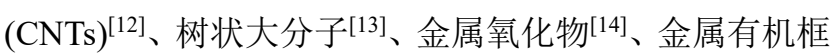
架 $(\mathrm{MOFs})^{[15]}$ 和二维过渡金属碳化物、氮化物或碳氮化

*E-mail: baiyunfeng1130@126.com; feng-feng64@263.net

Received December 22, 2020; published February 1, 2021.

Project supported by the National Natural Science Foundation of China (No. 21975146), the Natural Science Foundation of Shanxi Province (No. 201801D121035), Cultivate Scientific Research Excellence Programs of Higher Education Institutions in Shanxi Province (No. 2020KJ023) and Shanxi Scholarship Council of China (No. 2020-133).

项目受国家自然科学基金(No. 21975146)、山西省自然科学基金(No. 201801D121035)、山西省高等学校科学研究优秀成果培育项目(No. 2020KJ023) 和山西省省筹资金资助回国留学人员科研项目(No. 2020-133)资助. 
物(MXenes) ${ }^{[16]}$ 等. 然而, 这些载体普遍存在缺乏靶向释 放特性、不易从体内清除、释放药物的外界条件要求较 高等问题. 因此, 研制新型纳米药物载体用于癌症治疗 仍然有很大的发展空间.

共价有机框架(COFs) 是近年来开发的一种由轻质 元素(氢、碳、氮、氧、硼等)通过共价键连接而成的高 结晶性多孔有机聚合物 ${ }^{[17-18]}$, 它所具有的孔道结构规则 有序、功能多样、比表面积大和孔隙率高等特点使其成 为净化、催化、生物传感和纳米医学等领域中极具潜力 的工具 ${ }^{[19-27]}$. 在 2005 年, Yaghi 课题组 ${ }^{[28]}$ 通过缩合反应 首次合成了两种 $2 \mathrm{D}$ COFs: $\left(\mathrm{C}_{3} \mathrm{H}_{2} \mathrm{BO}\right)_{6}\left(\mathrm{C}_{9} \mathrm{H}_{12}\right)_{1}(\mathrm{COF}-1)$ 和 $\mathrm{C}_{9} \mathrm{H}_{4} \mathrm{BO}_{2}(\mathrm{COF}-5)$, 它们可在 $500 \sim 600{ }^{\circ} \mathrm{C}$ 下保持稳 定, 且比表面积可达到 711 和 $1590 \mathrm{~m}^{2} / \mathrm{g}$. 在 2007 年, 该 课题组 ${ }^{[29]}$ 又首次合成了两种 $3 \mathrm{D}$ COFs: COF-102 和 COF-103, 这些材料完全由 C-C、C-O 和 C-B 等构成. 近 年来, 在癌症治疗领域 COFs 因其各项优异的特性受到 研究者越来越多的关注, 特别是其具有良好的孔径可调 节性. 在 2015 年, Yan 课题组 ${ }^{[30]}$ 通过酰亚胺化反应合成 了两种 $3 \mathrm{D}$ COFs 首次用于载药, 这两种载药 COFs 均显 示出了较高的载药量(质量分数 $24 \%$ ) 和良好的缓释效 果. 2016 年, Lotsch 课题组 ${ }^{[31]}$ 将 TTI-COF 作为抗癌药物 檞皮素的载体, 这种通过氢键将 COFs 与药物结合的方 式突出了 $\mathrm{COFs}$ 作为纳米载体运输药物的潜力.

在这篇综述中, 我们总结了 COFs 的合成方法及其 在肿瘤治疗中的应用. 虽然 COFs 的制备及应用研究目 前还处于起步阶段并面临许多挑战, 但 COFs 巨大的潜 力势必会为未来肿瘤治疗和制药领域提供一些新的方 法和途径.

\section{COFs 的合成方法}

目前已报道多种 COFs 合成方法, 主要包括溶剂热 合成法、机械化学合成法、微波合成法、离子热合成法、 界面合成法、室温合成法和纳米尺度 COFs 的合成方法， 其中溶剂热合成法最为成熟, 其应用也最为广泛. 本综
述已将各种方法的优缺点进行了比较, 如表 1 所示.

\section{1 溶剂热合成法}

溶剂热合成法合成 COFs 的过程中, 反应条件与结 构单元的溶解度和反应的可逆性都有着密切的关系，同 时反应时间、温度、溶剂条件和催化剂浓度都是合成过 程中重要的影响因素 ${ }^{[32]}$. 目前, 人们已通过该方法合成 了多种 COFs.

Jiang 课题组 ${ }^{[33]}$ 合成了一种镍酞菁共价有机框架 $(\mathrm{NiPc} C \mathrm{COF}$ )(图 1A). 由于酞菁单元的有序堆叠, $\mathrm{NiPc}$ $\mathrm{COF}$ 表现出良好的光吸收率和电荷传输能力. 此外该 课题组 ${ }^{[34]}$ 合成的由方酸连接的 CuP-SQ COF(图 1B) 由于 结构中存在 $\pi$ 共轭键使其具有了优异的稳定性. Yan 课 题组 ${ }^{[35}$ 通过酰亚胺化反应制备了一系列孔径为 4.2 $\mathrm{nm} \times 5.3 \mathrm{~nm}$ 且表面积高达 $2346 \mathrm{~m}^{2} / \mathrm{g}$ 的聚酰亚胺 (PI)COFs(图 1C). 为了解决溶剂热合成过程可能会造成 环境污染的问题 ${ }^{[36]}$, Banerjee 课题组 ${ }^{[37]}$ 用水代替有机溶 剂成功合成出 $\beta$-酩烯胺 $\mathrm{COF}$ (图 1D), 开发了一条更加绿 色环保的 COFs 合成途径. 此外, 本课题组 ${ }^{[38]}$ 通过醛、 胺和单质硫之间的组装反应, 首次通过一锅法合成了 5 种噻唑类化合物(TZ-COF), 研究表明, 这些材料表现出 了高结晶度、高比表面积和优异的物理化学稳定性.

目前为止，溶剂热合成法是合成 COFs 最成熟的方 法, 它能够为反应系统提供充足能量的特性使得产物更 容易突破吉布斯自由能从而形成晶体 ${ }^{[39]}$. 但是它的缺 点也同样明显, 即合成过程中条件要求高, 反应时间长, 使得该方法难以用于 COFs 的大规模生产.

\section{2 机械化学合成法}

机械化学合成法能够以简单、绿色的途径构建化学 键, 与溶剂热合成法形成了鲜明的对比 ${ }^{[40-41]}$. Banerjee 课题组 $\left.{ }^{[2]}\right]$ 通过手动研磨的方式合成了由 $\beta$-酮胺连接的 $\mathrm{COFs}$ (图 2A), 并在合成过程中观察到 $\mathrm{COFs}$ 层的剥落和 COFs 的形成. 这些由机械化学法合成的 COFs 在酸、碱 和高温中仍可保持原有结构, 但结晶度略差. 为了解

表 1 COFs 合成方法优缺点比较

Table 1 Comparison of advantages and disadvantages of COFs synthesis method

\begin{tabular}{|c|c|c|}
\hline 合成方法 & 优点 & 缺点 \\
\hline 溶剂热合成法 & $\begin{array}{l}\text { 能够为反应系统提供充足能量的特性使得产物更容 } \\
\text { 易突破吉布斯自由能从而形成晶体 }\end{array}$ & $\begin{array}{l}\text { 合成过程中条件要求高, 反应时间长, 难以用于 } \\
\text { COFs 的大规模生产 }\end{array}$ \\
\hline 机械化学合成法 & $\begin{array}{l}\text { 能够以简单、绿色的途径构建化学键, } \\
\text { 合成的 COFs 在酸、碱和高温中仍可保持原有结构 }\end{array}$ & 结晶度略差 \\
\hline 离子热合成法 & 可以在常压下进行 & $\begin{array}{l}\text { 会导致材料部分碳化, 体系的能耗过高, 并且要求 } \\
\text { 单体必须具有较高的溶解性和热稳定性 }\end{array}$ \\
\hline 界面合成法 & 可以制备出结构良好的 COFs 薄膜 & 无法实现 COFs 的大规模生产 \\
\hline 室温合成法 & 对合成反应的温度要求较低, 易于操作 & $\begin{array}{l}\text { 只能用于制备少量席夫碱类的 COFs, 应用范围较 } \\
\text { 小 }\end{array}$ \\
\hline 纳米尺度 COFs 的合成方法 & 可有效控制 COFs 形貌 & 机理复杂, 操作繁琐 \\
\hline
\end{tabular}


A
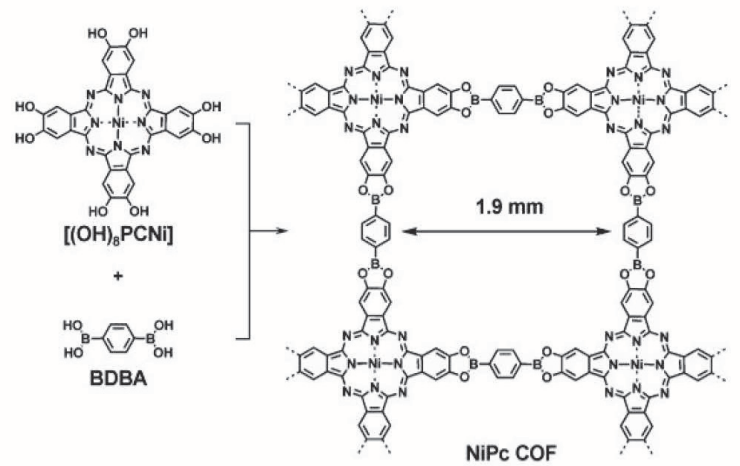

B
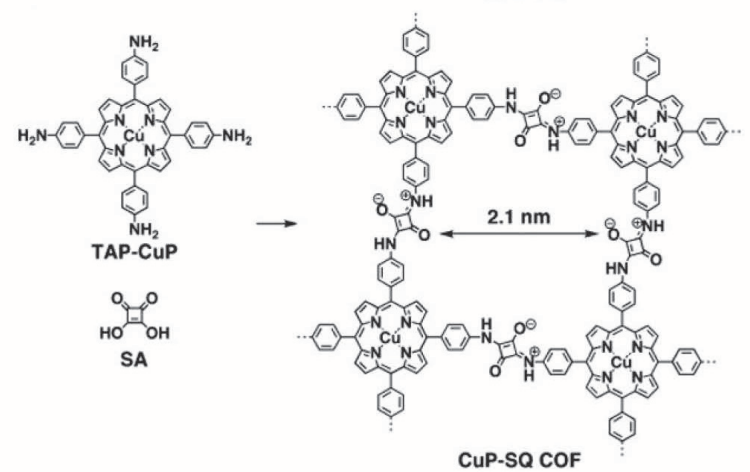

C

b

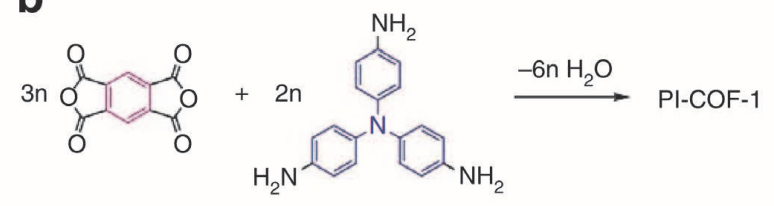

C<smiles>NCC(CN)C(CN)C(N)O</smiles>

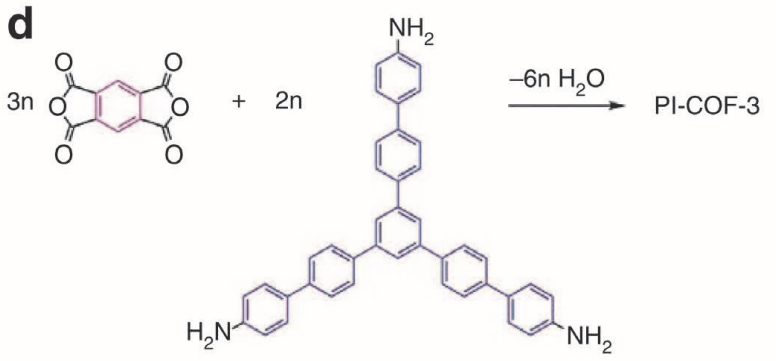

D

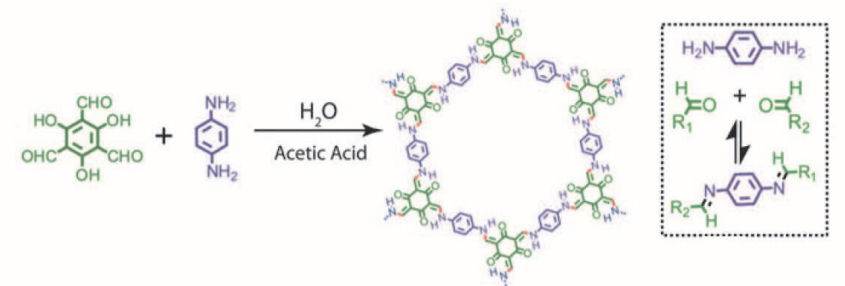

图 1 通过溶剂热合成法合成 COFs 的过程和结构: (A) NiPc COF 的合成和结构 ${ }^{[33]}$; (B) CuP-SQ COF 的合成和结构 ${ }^{[34]}$; (C) PI-COF-1, PI-COF-2 和 PI-COF-3 的合成和结构 ${ }^{[35]}$; (D) $\beta$-酮烯胺 COF 的合成和结构 ${ }^{[37]}$

Figure 1 Processes and structures of COFs synthesized by solvothermal method: (A) Synthesis and structure of NiPc COF ${ }^{[33]}$; (B) synthesis and structure of CuP-SQ COF ${ }^{[34]}$; (C) syntheses and structures of PI-COF-1, PI-COF-2 and PI-COF- ${ }^{[35]}$; (D) synthesis and structure of $\beta$-ketoenamine COF $^{[37]}$

决该问题, 该课题组 ${ }^{[43]}$ 开发了液体辅助研磨方法(图 2B), 该方法通过引入少量催化剂以提高合成反应速率, 改善 $\mathrm{COFs}$ 的结晶度.

\section{3 微波合成法}

在溶剂热合成法中晶体是在靠近壁面处生长, 所以 合成速度比较缓慢, 而在微波法中晶体可以在整个溶液 中快速生长，因此人们尝试使用微波法来合成 COFs. 2009 年, Cooper 课题组 $\left.{ }^{[4]}\right]$ 首次应用这种方法合成了 COF-5 (图 2C). 与传统方法合成的 COF-5 相比, 该 COFs 表现出了相似的性能, 但合成反应时间却被大大地缩 短, 比表面积也显著提高(由 $1590 \mathrm{~m}^{2} / \mathrm{g}$ 提高到 2019 $\mathrm{m}^{2} / \mathrm{g}$ ). 通过该方法, 已成功制备出各种新型 $\mathrm{COFs}$, 包 括 COF-102、BND-TFB COF 等 ${ }^{[44-47]}$. 微波合成法合成 速度快的特点使其成为一种理想的 COFs 合成方法, 但 此种方法对设备要求较高, 且会损耗大量能量.

\section{4 离子热合成法}

由于离子液体具有极低的熔点和蒸汽压, 离子热合
成过程可以在常压下进行. 2008 年, Thomas 课题组 ${ }^{[48]}$ 首 次通过离子热法合成了三嗪连接的 $\mathrm{COFs}(\mathrm{CTF})$ (图 2D). 研究表明, 这些材料具有较大的比表面积和孔体积. 离 子热合成法反应温度较高, 会导致材料部分碳化, 体系 的能耗过高, 并且要求单体必须具有较高的溶解性和热 稳定性，这极大限制了其适用范围，因此暂时还无法实 现 COFs 的大规模生产.

\section{5 界面合成法}

不同于其它只能合成 COFs 粉末的合成方法, 界面 合成法可以在界面处制备 COFs 薄膜并控制其厚度 ${ }^{[49-50]}$. 2017 年, $\mathrm{Xu}$ 课题组 ${ }^{[51]}$ 在二氯甲烷和三氟甲磺酸的界面 处成功制备了由三嗪单体构成的 COFs 膜(图 3A). 此外, $\mathrm{Li}$ 课题组 ${ }^{[52]}$ 通过 Suzuki 偶联反应合成了薄膜状的 2DCCOF1 和 2DCCOF2(图 3B). 不同于其它只能合成 COFs 粉末的方法, 界面合成法可以制备出结构良好的 COFs 薄膜, 但是目前通过该方法仍然无法实现 COFs 的大规模生产. 
A
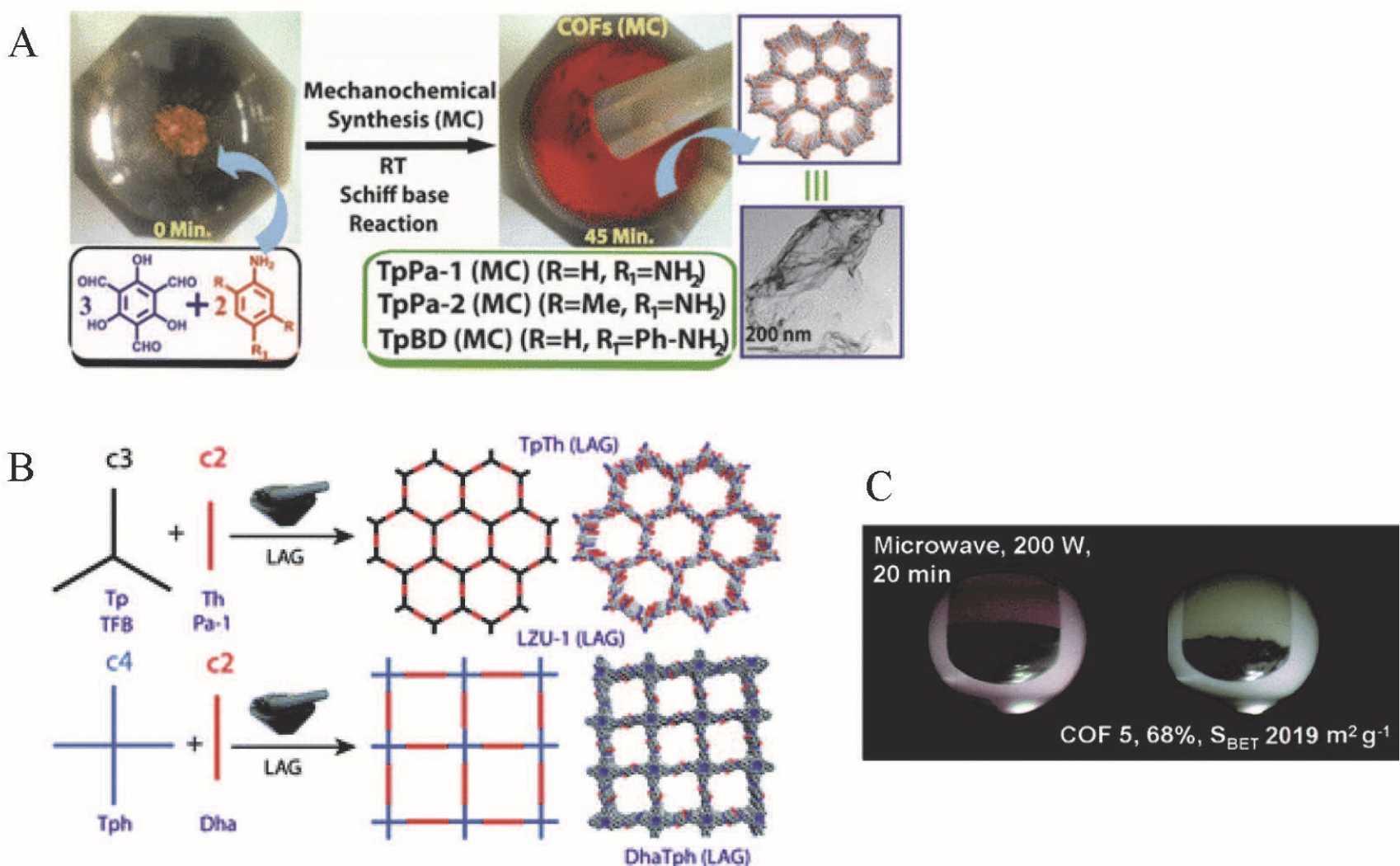

$\mathrm{D}$ b)

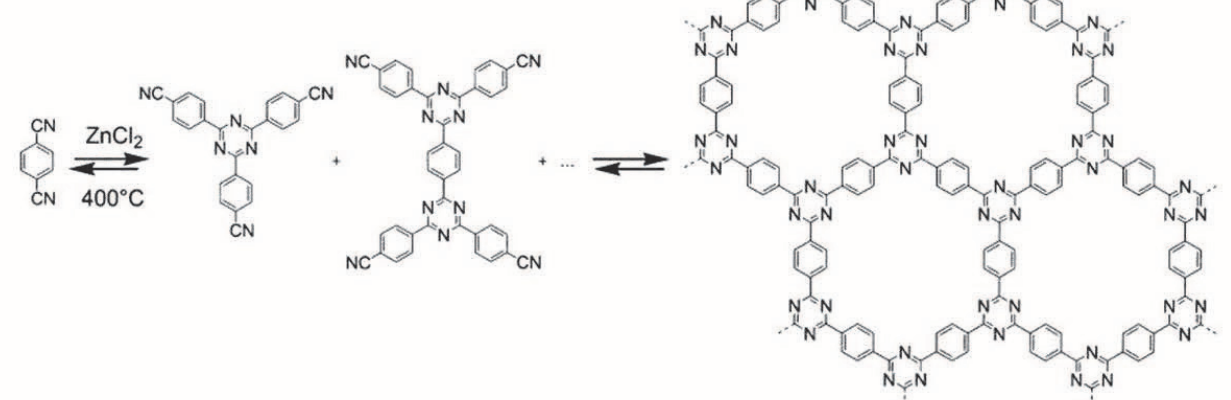

图 2 通过机械化学合成法、微波合成法和离子热合成法合成 COFs 的过程和结构: (A) COFs(MC)的合成和结构 ${ }^{[2]}$; (B) TpTh, LZU-1 和 DhaTph 的合成和结构 ${ }^{[43]} ;$ (C) 通过微波合成法合成 COF-5 ${ }^{[44]}$; (D) CTF 的合成和结构 ${ }^{[48]}$

Figure 2 Processes and structures of COFs synthesized by mechanochemical synthesis, microwave synthesis, and ionic thermal synthesis: (A) Syntheses and structures of COFs (MC ${ }^{[42]}$; (B) syntheses and structures of TpTh, LZU-1 and DhaTph ${ }^{[43]}$; (C) synthesis of COF-5 by microwave synthesis ${ }^{[44]}$; (D) synthesis and structure of $\mathrm{CTF}^{[48]}$

\section{6 室温合成法}

由于溶剂热合成法对温度要求较高, 体系能耗较 大, 研究者们尝试在室温条件下合成 COFs. Wang 课题 组 ${ }^{[33-54]}$ 在 2013 年首次提出室温合成法并在 2017 年将其 成功用于 COFs 合成(图 3C). 单体被溶解后放置在乙酸 中进行催化, 在室温条件下静置 $3 \mathrm{~d}$, 最终得到了 $\mathrm{COFs}$ 沉淀. 此外, Bein 课题组 ${ }^{[55]}$ 使用类似的方法制备出 BDT-COF 和 COF-5(图 3D). 室温合成法是一种新颖的 合成方法, 它对合成反应的温度要求较低, 易于操作, 但是目前只能用于制备少量席夫碱类的 COFs, 应用范 围较小.

\section{7 纳米尺度 COFs 的合成方法}

已报道的合成方法中, 得到的 COFs 尺度大多在微 米级, 与体细胞体积不相上下, 导致其难以应用于生物 医学领域. 目前纳米尺度 COFs 的合成方法主要分为两 类，即自上而下的方法和自下而上的方法 ${ }^{[56]}$.

自上而下的方法即是通过破坏 COFs 的层间作用力 得到纳米尺度 COFs 薄片. 2011 年, Zamora 课题组 ${ }^{[57]}$ 通 过将合成的 COF-8 超声剥离得到了厚度为 $4 \sim 10 \mathrm{~nm}$ 、 横向尺寸为几微米的 COF-8 纳米片(图 4A). 2013 年, Banerjee 课题组 ${ }^{[58]}$ 首次通过机械剥离法制得厚度在 3 $10 \mathrm{~nm}$ 之间的 COFs 薄片(图 4B). 此外, 纳米尺度的 
A

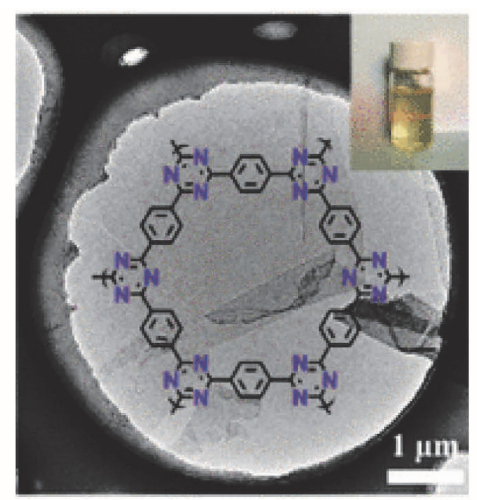

$\mathrm{C}$

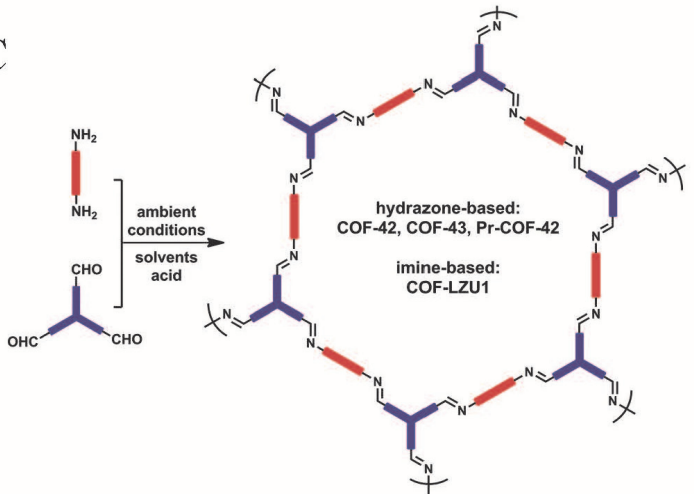

B

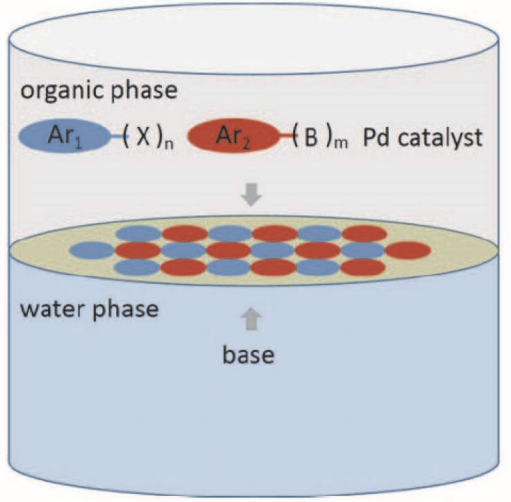

$\mathrm{D}$

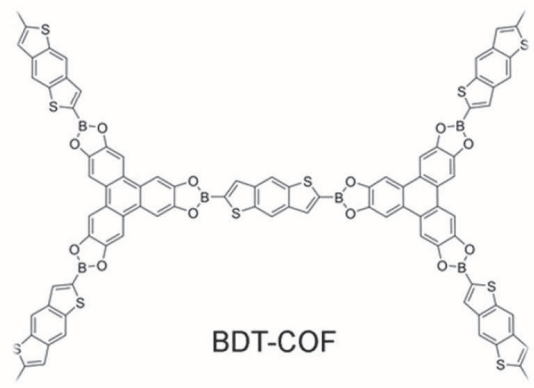

图 3 通过界面合成法和室温合成法合成 COFs 的过程和结构: (A)由三嗪单体组成的 $\mathrm{COFs}^{[51]}$; (B) $2 \mathrm{DCCOF} 1$ 和 $2 \mathrm{DCCOF} 2$ 的合成 ${ }^{[52]}$; (C) COF-42, COF-43, Pr-COF-42 和 COF-LZU1 的合成和结构 ${ }^{[54]}$; (D) BDT-COF 的结构 ${ }^{[5]}$

Figure 3 Processes and structures of COFs synthesized by interface synthesis and room temperature synthesis: (A) COFs composed of triazine monomers $^{[51]}$; (B) syntheses of 2DCCOF1 and 2DCCOF2 ${ }^{[52]}$; (C) syntheses and structures of COF-42, COF-43, Pr-COF-42 and COF-LZU1 ${ }^{[54]}$; (D) structure of BDT-COF ${ }^{[5]}$

A

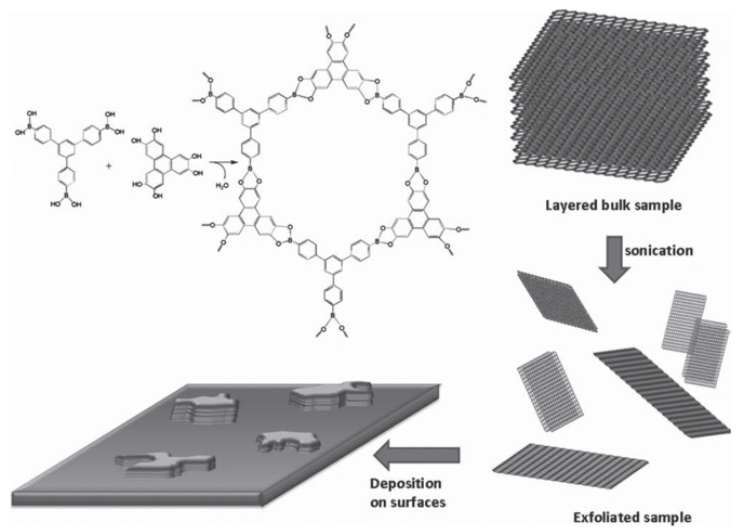

$\mathrm{C}$

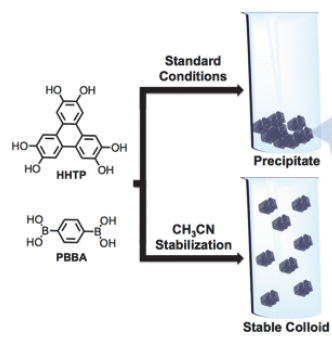

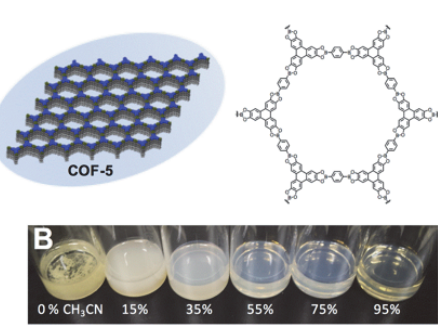

$\mathrm{B}$

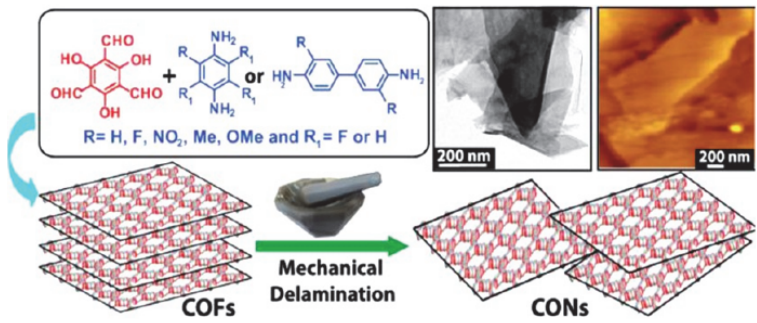

$\mathrm{D}$

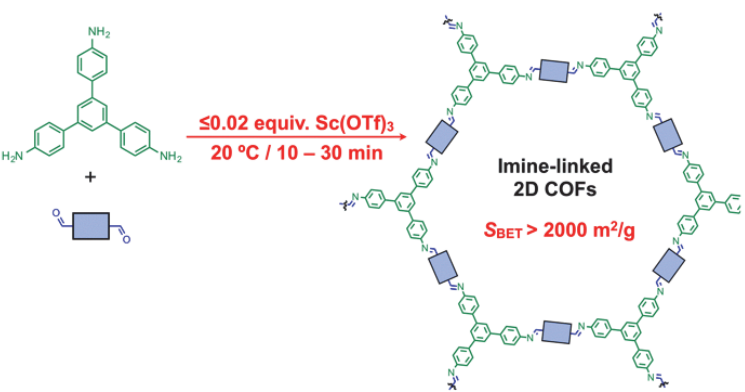

图 4 纳米尺度 COFs 的合成过程和结构: (A)纳米尺度 COF-8 的合成和结构 ${ }^{[57]}$; (B)纳米尺度席夫碱类 COFs 的合成和结构 ${ }^{[58]}$; (C)纳米尺度 COF-5 的合成和结构 ${ }^{[62]}$; (D) 纳米尺度 TTA-AzoDFP 的合成和结构 ${ }^{[63]}$

Figure 4 Synthesis processes and structures of nanoscale COFs: (A) Synthesis and structure of nanoscale COF- ${ }^{[57]}$; (B) syntheses and structures of nanoscale Schiff $\mathrm{COFs}^{[58]}$; (C) synthesis and structure of nanoscale COF-5 ${ }^{[62]}$; (D) synthesis and structure of nanoscale TTA-AzoDFP ${ }^{[63]}$ 
TP-Por COFs、TpBD COF、NUS 30-32 等也已成功通过 自上而下的方法合成 ${ }^{[59-61]}$. 自上而下的方法适用性广、 效果好, 但是操作过程耗时耗能, 且所得纳米片形貌不 均匀.

自下而上的方法是通过单体直接合成纳米尺度 COFs. 受乙腈可稳定胶体的启发, 2017 年, Dichtel 课题 组 ${ }^{[62]}$ 通过加入乙腈成功合成了纳米尺度的 COF-5, 研究 表明, 当乙腈体积含量达到 95\%时, COF-5 的粒径可缩 小至 $50 \mathrm{~nm}$ (图 4C). 2019 年, Trabolsi 课题组 ${ }^{[63]}$ 通过缩短 反应时间抑制晶体的生长过程成功合成了纳米尺度的 TTA-AzoDFP(粒径仅为 $117 \mathrm{~nm}$ )(图 4D). 此外还通过自 下而上的方法制得纳米尺度的 TAPB-PDA COF、 $\mathrm{COF}-42$ 等 $\mathrm{COFS}^{[64-66]}$. 自下而上的方法所得产品形貌较 为均匀且更易控制, 但是机理复杂、操作繁琐、适用性 较窄.

\section{COFs 在肿瘤治疗中的应用}

近年来, COFs 由于具有密集孔结构、低毒性等特点 被广泛用于纳米载药系统研究. COFs 充当载体将药物
递送至靶目标或是利用自身具有的光热或光动力效应 对癌细胞进行杀伤在肿瘤治疗中取得了显著的效果. COFs 在肿瘤治疗中的应用可以划分为药物化疗、光热 疗法、光动力疗法以及联合疗法四种, 而其中联合疗法 由于具有多种作用方式对肿瘤有着较好的杀伤效果，应 用前景广泛.

\section{1 药物化疗}

COFs 由于规则的孔结构和较大的比表面积具有出 色的负载能力, 并且可以在到达目标前保持稳定结构并 在完成输送任务后进行降解, 因此在药物化疗中通常被 用作载体将药物递送至靶目标.

2016 年, Zhao 课题组 ${ }^{[67]}$ 合成了两种亚胺类 2D COFs (PI-3-COF 和 PI-2-COF)(图 5A)并研究了它们的载 药效果. 研究证明, 这些 2D COFs 具有良好的生物相容 性, 而且在水中依然能保持良好的孔隙结构并以纳米粒 子的形式稳定存在. 该课题组使用 5-氟尿嘧啶(5-FU)、 卡托普利和布洛芬(IBU)三种不同药物对 COFs 的药物 加载和释放进行研究, 并采用粉末 $\mathrm{X}$ 射线衍射(PXRD)、 热重分析(TGA)和傅立叶变换红外光谱(FTIR)表征载药

A

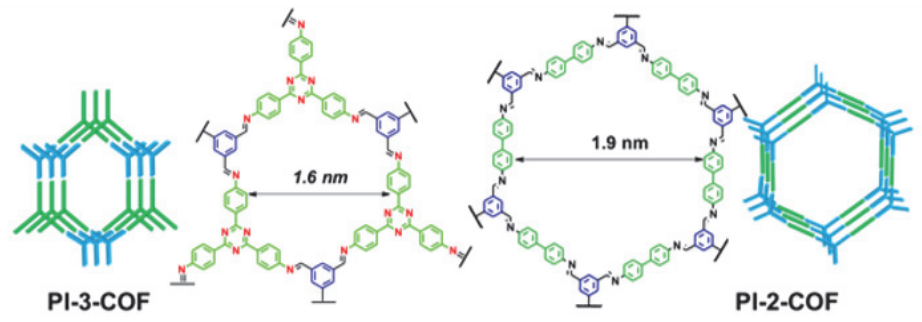

B

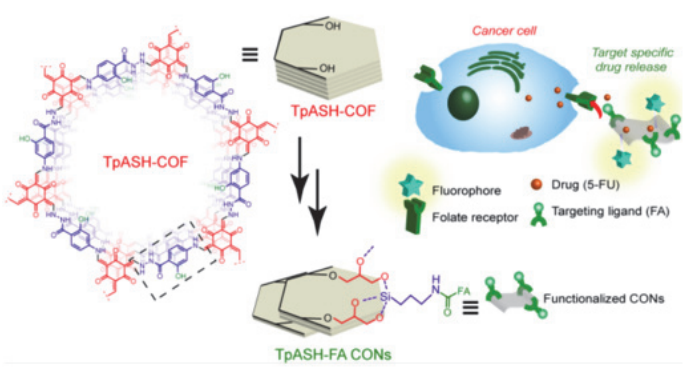

D

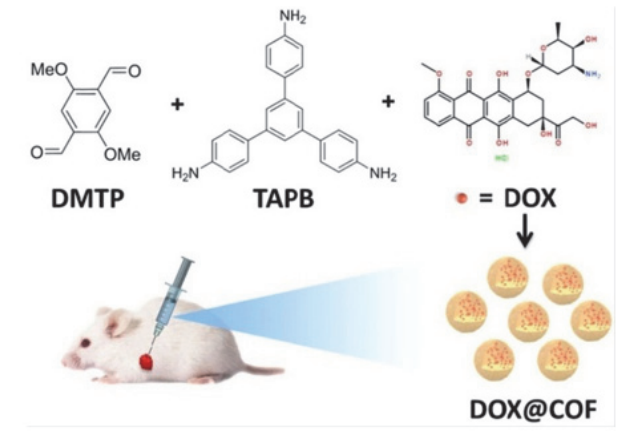

$\mathrm{C}$

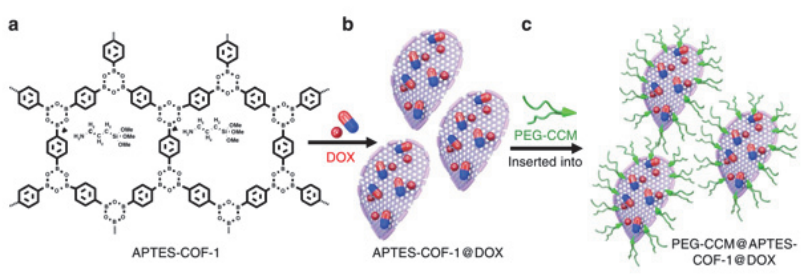

d

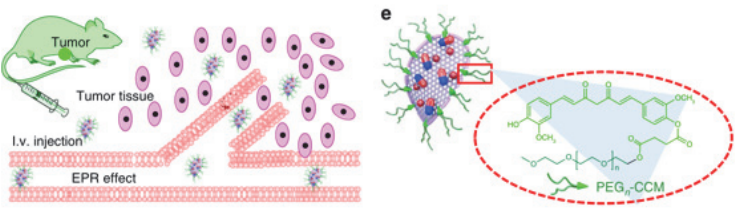

图 5 COFs 在药物化疗中的几种应用: (A) PI-3-COF 和 PI-2-COF 的结构 ${ }^{[67]}$; (B) TpASH-FA 在肿瘤治疗中的应用 ${ }^{[68]}$; (C) PEG-CCM@APTES-COF-1 在肿瘤治疗中的应用 ${ }^{[69]}$; (D) DOX@TAPB-DMTP-COF 在肿瘤治疗中的应用 ${ }^{[70]}$

Figure 5 Several applications of COFs in chemotherapy: (A) Structures of PI-3-COF and PI-2-COF ${ }^{\text {[67]; }}$ (B) application of TpASH-FA in tumor therapy $^{[68]} ;(\mathrm{C})$ application of PEG-CCM@APTES-COF-1 in tumor therapy ${ }^{[69]}$; (D) application of DOX@TAPB-DMTP-COF in tumor therapy ${ }^{[70]}$ 
COFs 以及采用 TGA 测定了 COFs 的载药效果(载药量高 达 30\%). 细胞实验表明, 经载药系统处理的 MCF7 细胞 存活率显著降低.

正如其他载体一样, 单纯的 COFs 载药系统不具有 癌细胞靶向性. 2017 年, Banerjee 课题组 ${ }^{[68]}$ 合成了两种 COFs (TpASH 和 TpAPH), 并选择 TpASH 进行修饰, 制 备出用于靶向的叶酸共轭共价有机纳米片(CONs)(图 $5 \mathrm{~B})$. 这种靶向 CONs 可以通过受体介导的内吞作用将 药物 5-FU 输送到乳腺癌细胞并将其杀死. UV-Vis 分析 显示, 该载药系统上负载了 $12 \%$ 的 $5-F U$. 随后通过 MTT 实验和苂光显微镜分别进行了细胞迁移和细胞摄 取研究, 结果表明该系统具有良好的癌细胞靶向性. 虽 然这种系统载药量较低, 但是对乳腺癌细胞的成功靶向 研究使其在靶向给药方面拥有了巨大的潜在价值.

然而, 未经修饰的 COFs 在体内水分散性较差, 为 改善此问题, 2018 年, Jia 课题组 ${ }^{[69]}$ 报道了一种胺基化 COF-1 的载药系统(图 5C). 该课题组通过将聚乙二醇修 饰的姜黄素衍生物 (PEG-CCM) 和胺化 COFs (APTES-COF-1)进行组装, 制备了一系列具有水分散性 的 COFs 纳米复合材料, 并最终组成了负载抗癌药物阿 需素 $(\mathrm{DOX})$ 的 COFs 载药系统. 该系统可看作是以 APTES-COF-1 为油相, PEG-CCM 为表面活性剂的胶束. 系统到达细胞内部后, PEG-CCM 会脱落, 从而释放出 DOX. 该项研究中, 作者使用 PEG-CCM 对 COFs 进行 表面改性, 显著提高了载药系统的水解稳定性、载药量 和药物包封率, 使其能与最好的 MOFs 载药系统相婫美.

为探究更简单的 COFs 与药物的组装过程, 2019 年, Lin 课 题 组 ${ }^{[70]}$ 通 过一锅法成 功将 DOX 和 TAPB-DMTP-COF 组装成 COFs 载药系统(图 5D). 在组 装过程中, 作者先将 DOX 和 DMTP 混合搅拌 $1 \mathrm{~h}$, 然后 加入 TAPB 试剂, 最终通过席夫碱反应形成了 DOX@COF. 作者利用扫描电子显微镜(SEM)检测到当 DOX 负载到 COFs 中后形成了尺寸在 $200 \sim 400 \mathrm{~nm}$ 之间 的不均匀颗粒; 通过傅里叶红外光谱仪(FTIR)结果显示 出 COFs 与 DOX 具有良好的结合性; 根据 DOX 在紫 外-可见分光光度计 (UV-Vis) 光谱中的吸收强度测定出 该系统载药量约为质量分数 $32.1 \%$, 这个数值远高于此 前发表的结果; 此外还采用瘤内注射的方式在小鼠上进 行了体内实验, 结果表明该系统对癌细胞具有良好的杀 伤效果.

\section{2 光热疗法}

在过去的几年中, 利用光热疗法(PTT)进行癌症治 疗已然成为一种全新的方向. PTT 是利用光热转化剂 (PTA)在光照下产生的热量损伤肿瘤细胞 ${ }^{[71]}$. 在光热疗 法中, COFs 可充当修饰物提高纳米系统的光热效果或 本身作为光热剂, 还可以充当载体递送光热剂.

不同于阻碍热量传递的封闭多孔系统, COFs 可以 通过开放的孔隙通道将产生的热量快速传输到环境中,
促进水中的热传导，从而快速改变系统温度 ${ }^{[72]}$. 2016 年, Wang 课题组 ${ }^{[66]}$ 首次评估了纳米 COFs 用于光热治疗的 潜力. 该项研究首先以 $\mathrm{Fe}_{3} \mathrm{O}_{4}$ 为模板制备了核壳结构的 $\mathrm{Fe}_{3} \mathrm{O}_{4} @ \mathrm{COF}(\mathrm{TpBD})$, 然后用聚乙二醇进行修饰以提高 系统在溶液中的稳定性(图 6A). 表征结果显示, TpBD 的比表面积为 $1346 \mathrm{~m}^{2} / \mathrm{g}$ 、孔径大小在 $1.3 \sim 2.0 \mathrm{~nm}$ 之间、 壳层厚度为 $100 \mathrm{~nm}$. 光热性能的研究显示, 包含 COFs 的 $\mathrm{TpBD}$ 在 $785 \mathrm{~nm}$ 处的光热转换效率为 $21.5 \%$, 这比单 独使用 $\mathrm{Fe}_{3} \mathrm{O}_{4}$ 纳米簇的光热转换效率高 $2 \sim 3$ 倍.

阳离子自由基 COFs 由于 $\pi$ 耦合多层膜之间存在电 荷转移而具有优异的光物理性质，即通过非辐射弛豫实 现近红外吸收和光热转换, 因此该种 COFs 本身即可作 为光热剂. 基于这项性质, Guo 课题组 ${ }^{[73]}$ 制备了一种具 有光热性质的 $\mathrm{Py}-\mathrm{BPy}{ }^{+}-\mathrm{COF} / \mathrm{PEG}$ (图 6B). 研究发现, 该系统在 808 和 $1064 \mathrm{~nm}$ 激光照射下, 光热转换效率分 别高达 $63.8 \%$ 和 $55.2 \%$.

\section{3 光动力疗法}

光动力疗法(PDT)通过光敏剂(PSS)吸收光能后将 氧 $\left(\mathrm{O}_{2}\right)$ 激发为活性氧 $(\mathrm{ROS})$ 来杀死癌细胞 ${ }^{[74]}$. 目前, 光 动力疗法(PDT)的相关研究已被广泛关注, 部分研究成

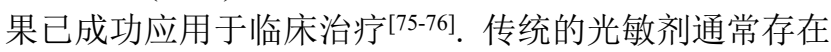
疏水性较差且容易 $\pi-\pi$ 堆积的问题, 导致了材料对靶组 织的选择性及 ROS 生成效果差等问题, 而 COFs 由于具 有易修饰和较大比表面积等特性有效缓解了这些问 题 ${ }^{[77]}$, 此外 COFs 由于具有扩展的共轭结构和高度有序 的 $\pi-\pi$ 堆积作用在激光照射下可产生单线态氧 ${ }^{[78]}$, 因此 在光动力疗法中, COFs 本身即可作为 PDT 治疗剂.

Dong 课题组 ${ }^{[79]}$ 首次将 COFs 用于光动力治疗, 作者 将 COFs 中的-CHO 与有机光敏剂嗍-二吡咯亚甲基 (BODIPY)进行席夫碱反应制得两种纳米尺度 COFs (LZU-1-BODIPY-2I 和 LZU-1-BODIPY-2H)(图 7A), 研 究表明, LZU-1-BODIPY-2I 表现出更强的细胞毒性.

为研究可通过肾脏过滤从体内清除的超小 COFs 纳 米点. $\mathrm{Qu}$ 课题组 ${ }^{[80]}$ 合成了一种基于卟啉的 COFs 纳米点. 为了改善其生理稳定性和生物相容性, 课题组通过聚乙 二醇(PEG)对 COFs 纳米点进行了修饰. 由于该系统具 有 3 4 nm 的超小尺寸，可以通过肾脏过滤从体内清除， 因此在体内不会引起明显的长期毒性; 而且卟啉的存在 使 COFs 纳米点可以在 $638 \mathrm{~nm}$ 光照射下有效产生 $\operatorname{ROS}($ 图 7B).

为探究具有更好 PDT 疗效的 COFs. Deng 课题组 ${ }^{[81]}$ 合成了两种具有 PDT 疗效的 COFs (COF-808 和 COF-909), 所合成的 COFs 具有稳定的孔隙率和出色的 光稳定性, 可促进氧气在癌细胞中的扩散和 ROS 的释 放, 拥有出色的 PDT 疗效(图 7C). 研究表明, 两种 COFs 可以在 $630 \mathrm{~nm}$ 激光照射下有效地生成 ROS 且 COF-909 表现出 COF-808 三倍的效果. 为了探究 COFs 的治疗效 果，课题组分别进行了体内和体外实验，结果表明 
COF-909 可通过内吞作用进入细胞且在 $630 \mathrm{~nm}$ 激光照 射下产生的 ROS 可有效抑制肿瘤组织的生长.

PDT 疗法中缺氧的肿瘤微环境会对治疗效果造成 显著的影响. Lin 课题组 ${ }^{[82]}$ 以 COFs 为模板, 在其表面逐

A

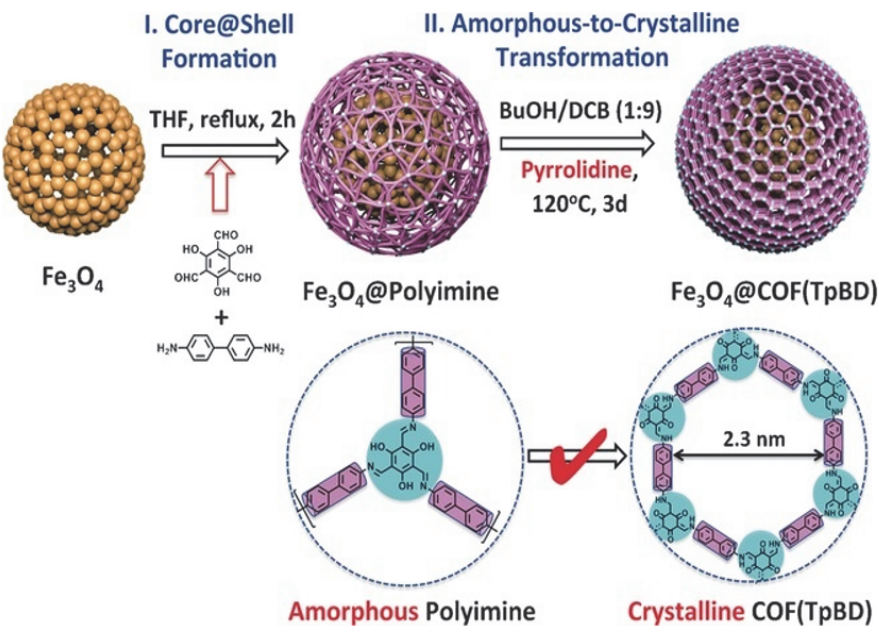

步修饰金纳米颗粒 $(\mathrm{Au} \mathrm{NPs})$ 、二氧化锰 $\left(\mathrm{MnO}_{2}\right)$ 与透明质 酸(HA)，生成 COF-Au-MnO $2-\mathrm{HA}$ 结构. 其中 $\mathrm{Au} \mathrm{NPs}$ 能 分解葡萄糖产生氧气从而改善影响 PDT 疗效的缺氧肿 瘤微环境, $\mathrm{MnO}_{2}$ 能消耗细胞内的谷胱甘肽 $(\mathrm{GSH})$ 增强抗

B

图 $6 \mathrm{COFs}$ 在光热疗法中的几种应用: (A) $\mathrm{Fe}_{3} \mathrm{O}_{4} @ \mathrm{COF}(\mathrm{TpBD})$ 在肿瘤治疗中的应用 ${ }^{[66]}$; (B) Py- $\mathrm{BPy}^{+}{ }^{+}-\mathrm{COF} / \mathrm{PEG}$ 在肿瘤治疗中的应用 ${ }^{[73]}$

Figure 6 Several applications of COFs in photothermal therapy: (A) Application of $\mathrm{Fe}_{3} \mathrm{O}_{4} @ \mathrm{COF}$ (TpBD) in tumor therapy ${ }^{[66]}$; (B) application of $\mathrm{Py}^{-\mathrm{BPy}^{+}}{ }^{+}-\mathrm{COF} / \mathrm{PEG}$ in tumor therapy ${ }^{[73]}$

A

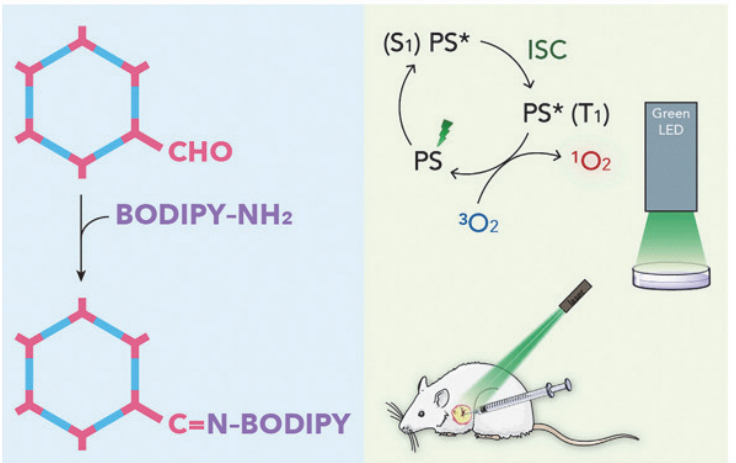

$\mathrm{C}$

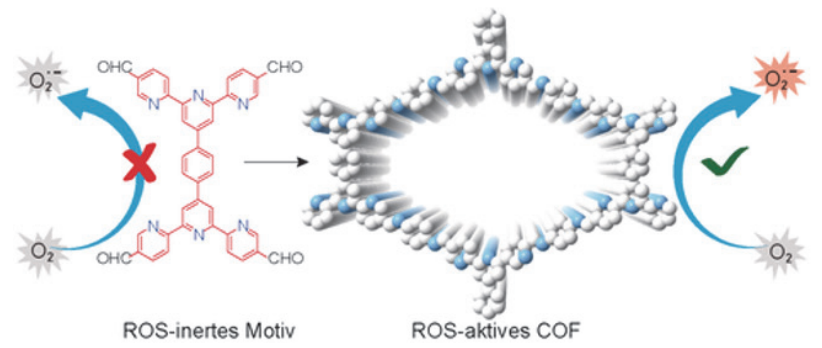

$\mathrm{B}$

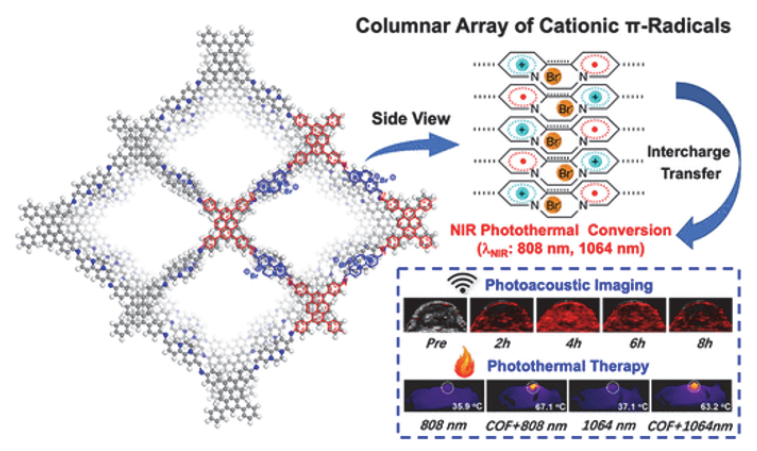

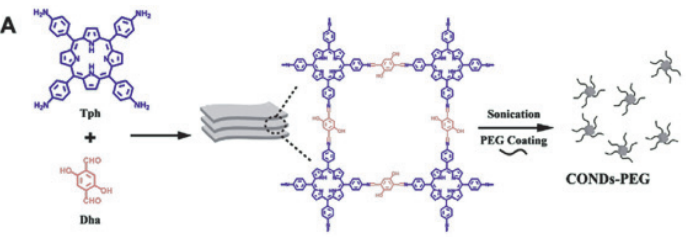

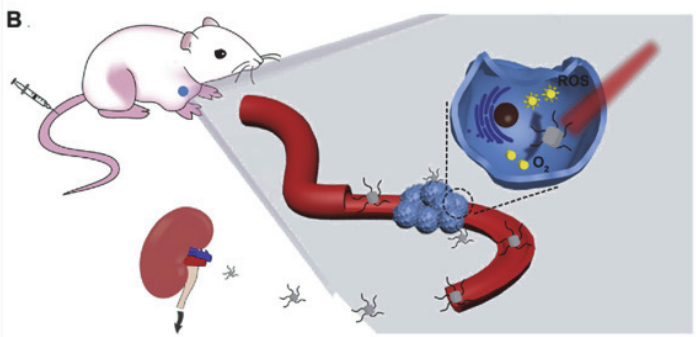

$\mathrm{D}$

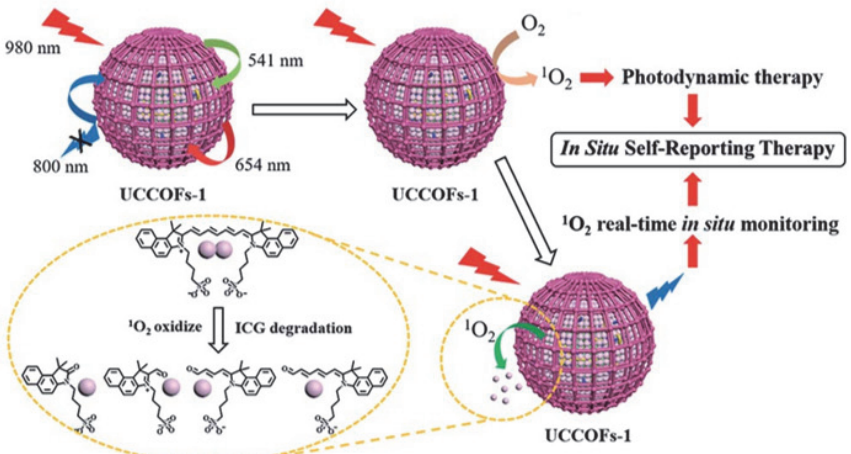

图 7 COFs 在光动力疗法中的几种应用: (A) LZU-1-BODIPY-2I 和 LZU-1-BODIPY-2H 在肿瘤治疗中的应用[79]; (B)基于卟啉的 COFs 纳米点在肿 瘤治疗中的应用 ${ }^{[80]}$; (C) COF-909 在肿瘤治疗中的应用 ${ }^{[81]}$; (D) UCCOFs-1 在肿瘤治疗中的应用 ${ }^{[83]}$

Figure 7 Several applications of COFs in photodynamic therapy: (A) Application of LZU-1-BODIPY-2I and LZU-1-BODIPY-2H in tumor therapy ${ }^{[79]}$; (B) application of COFs nanodots based on porphyrin in tumor therapy ${ }^{[80]}$; (C) application of COF-909 in tumor therapy ${ }^{[81]}$; (D) application of UCCOFs-1 in tumor therapy ${ }^{[83]}$ 
肿瘤作用, 研究表明, 低氧环境下, 经 COF-Au$\mathrm{MnO}_{2}-\mathrm{HA}$ 处理的肿瘤细胞在激光照射下调亡率可达 $15.2 \%$.

此外, Tan 课题组 ${ }^{[83]}$ 基于核介导的方法开发出一种 能够产生高效近红外辐射和 ROS 的 “上转换 COF 纳米 系统” (UCCOFs) 并通过调整 COFs 壳层的厚度使 COFs 的光动力效率提高了 12.5 倍(图 7D). 研究发现, 系统在 $980 \mathrm{~nm}$ 近红外光的照射下有效生成了 ROS.

\section{4 联合治疗}

现在人们已经发现单一疗法治疗癌症的效果并不 理想. 例如, 光敏剂在缺氧的肿瘤微环境下发生自我猝 灭会阻碍 ROS 的释放 ${ }^{[84-87]}$, 导致治疗效率低下. 然而 COFs 可以与多种组分结合并进行修饰形成纳米载药系 统从而进行各种联合疗法, 获得更好的治疗效果. 目前 在联合治疗中, 光热与光动力联合疗法应用最为广泛且 疗效最好.

\subsection{1 光热与光动力联合疗法}

COFs 具有多种功能的官能团以及较大的内部通 道 ${ }^{[88]}$, 可以通过化学官能化或封装策略来包裹光敏剂 和光热剂分子，从而实现 PDT/PTT 的联合效应 ${ }^{[89]}$.

近年来, 吲哚菁绿(ICG)作为光热剂和光敏剂已广 泛应用于肿瘤治疗. Yuan 课题组 ${ }^{[90]}$ 首先将 ICG 吸附到块 状 COF-1 上, 然后通过超声剥离得到了 ICG@COF-1 纳 米片, 最后将同样具有光热效应的聚多巴胺(PDA)和修 饰物聚乙二醇(PEG)依次修饰到 ICG@COF-1 的表面上, 进一步提高了该系统在体内的稳定性和循环时间. 研究 表明, 系统在 $808 \mathrm{~nm}$ 激光照射下会产生大量的热量和 $\operatorname{ROS}$ (图 8A), 表现出明显的光热与光动力联合疗效.

Chen 课题组 ${ }^{[59}$ 选择卟啉单体制备了三苯基卟啉 (TP-POR) COF, 并通过液体超声分层剥离成直径为 345 $\mathrm{nm}$ 的 CONs. 研究表明, 在 $635 \mathrm{~nm}$ 激光照射下, CONs 可产生活性氧并具有 $19.1 \%$ 的光热转化率, 可诱导 $18.8 \%$ 的 HeLa 细胞坏死. 此外, Guo 课题组 ${ }^{[91]}$ 通过类似 的方法制备粒径约为 $100 \mathrm{~nm}$ 的 COF-366, 并将该系统 应用于光热与光动力联合疗法.

Dong 课题组 ${ }^{[92]}$ 通过客体封装的方式将光敏剂卟啉 (Por)和光热剂菜酞菁 PTA (VONc)组装到合成的纳米尺 度 COFs (NCOF)上, 开发出一种理想的 PDT/PTT 联合 治疗系统(图 8B). 研究表明, 在可见光(红色 LED)和近 红外激光 $(808 \mathrm{~nm})$ 的照射下, 系统能够产生大量的 ROS 并达到 $55.9 \%$ 的光热转化率; 体外和体内实验均表明, 该系统可有效控制 MCF7 肿瘤细胞的增殖和转移.

Pang 课题组 ${ }^{[93]}$ 首先通过液相合成法在室温下制备 了高度单分散的 COFs 纳米粒子(COF-LZU-1), 然后将 COF-LZU-1 直接与 $\mathrm{Cu}\left(\mathrm{NO}_{3}\right)_{2}$ 水溶液混合形成 COF$\mathrm{Cu}^{2+}$ 配合物, 最后加入新制备的 $\mathrm{Se}^{2-}$ 水溶液合成了 $\mathrm{COF}-\mathrm{CuSe}$ 纳米复合材料. 为了提高纳米颗粒的溶解度
和稳定性，作者又用 $\mathrm{NH}_{2}$-PEG-2000-COOH 对其进行修 饰(图 8C). 研究表明, 系统在 $808 \mathrm{~nm}$ 激光照射下达到了 $26.34 \%$ 的光热转换率并释放出 $\mathrm{ROS}$; 体外和体内实验 均表明系统表现出理想的联合疗效. 之后, 该课题组使 用 1,3,5-三(4-氨基苯基)苯(TAPB) 和 1,3,5-苯三醛 (BTCA) 合成了一种新型 COFs, 并将其与 ICG 偶联形成 COF@ICG (CI)(图 8D). 为了提高 CI 在体内的生物相容 性和产生 ROS 的能力, 作者将鸡卵清蛋白(OVA)涂覆在 CI 表面形成了COF@ICG@OVA (CIO). 研究表明, 在 $650 \mathrm{~nm}$ 和 $808 \mathrm{~nm}$ 激光照射下, CIO 表现出 $35.75 \%$ 的光 热转换效率和优异的 $\mathrm{ROS}$ 生成能力 ${ }^{[78]}$, 此外, TAPB-DMBP-COF 也被应用于类似的研究 ${ }^{[94]}$.

\section{4 .2 热疗与药物化疗联合疗法}

花青素(IR783)由于对近红外区域的光有良好的吸 收性并且生物相容性良好，近年来受到了广泛关注. 2019 年, Chen 课题组 ${ }^{[95]}$ 合成了一种新型 COFs (TP-Por $\mathrm{COF}$ )并通过超声剥离技术将 COFs 剥离成负载 IR783 的 COFs 纳米片(COF@IR783), 之后他们在该基础上进一 步加载了顺式-乌头酸酐修饰的阿霉素(CAD), 组成了 光热药物化疗联合治疗系统(图 9A). 通过体外细胞实验 发现, COFs 不影响细胞活力但系统对肿瘤细胞具有明 显的联合杀伤效果. 之后小鼠实验中发现, 经过该系统 处理的小鼠肿瘤组织明显坏死.

\subsection{3 光动力与药物化疗联合疗法}

Zhang 课题组 ${ }^{[96]}$ 将抗纤维化药物吡非尼酮(PFD)与 $\mathrm{COF}_{\text {TTA-DHTA }}$ 结合, 并用两亲聚合物聚(乳酸-乙醇酸)-聚 乙二醇 (PLGA-PEG) 进行修饰, 合成了

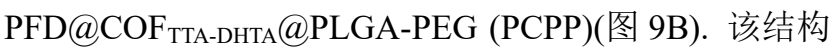
中, PCPP 在肿瘤区域释放的 PFD 可以破坏肿瘤的细胞 外基质(ECM), 从而促进肿瘤细胞对随后注射的光敏剂 原卟啉肽偶联纳米胶束(NM-PPIX)的摄取, 并增强对肿 瘤的 PDT 疗效.

细胞内 $\mathrm{Ca}^{2+}$ 含量过多会导致细胞死亡，其浓度在 细胞内受到严格控制，而这种控制很容易被 ROS 破坏， 因此理论上可通过 PDT 与加入外源 $\mathrm{Ca}^{2+}$ 的联合作用促 使细胞死亡. 受此机理启发, Dong 课题组 ${ }^{[97]}$ 报道了一种 基于纳米共价有机框架 (NCOF) 的纳米结构 $\left(\mathrm{CaCO}_{3} @\right.$ COF-BODIPY-2I@GAG)(图 9C). 该结构用碘代氟嗍二 吡咯(BODIPY2I) 作为光敏剂和 $\mathrm{CaCO}_{3}$ 作为提供外源 $\mathrm{Ca}^{2+}$ 的物质, 研究表明, 系统在光照下产生的 ROS 不仅 可以直接杀死肿瘤细胞, 还可以导致肿瘤细胞内 $\mathrm{Ca}^{2+}$ 含量升高从而杀死细胞, 显示出显著的抗肿瘤效果.

\subsection{4 磁性热疗与药物化疗联合疗法}

Trabolsi 课题组 ${ }^{[98]}$ 通过纳米尺度的 TAB-DFP-nCOF 负载 DOX, 之后分别修饰磁性氧化铁纳米颗粒 $\left(\gamma-\mathrm{Fe}_{2} \mathrm{O}_{3}\right.$ NPs) 和聚赖氨酸阳离子聚合物(PLL), 制得平均粒径为 $300 \mathrm{~nm}$ 的系统 $(\gamma-\mathrm{SD} / \mathrm{PLL})$, 在交变磁场 (AMF) 中 


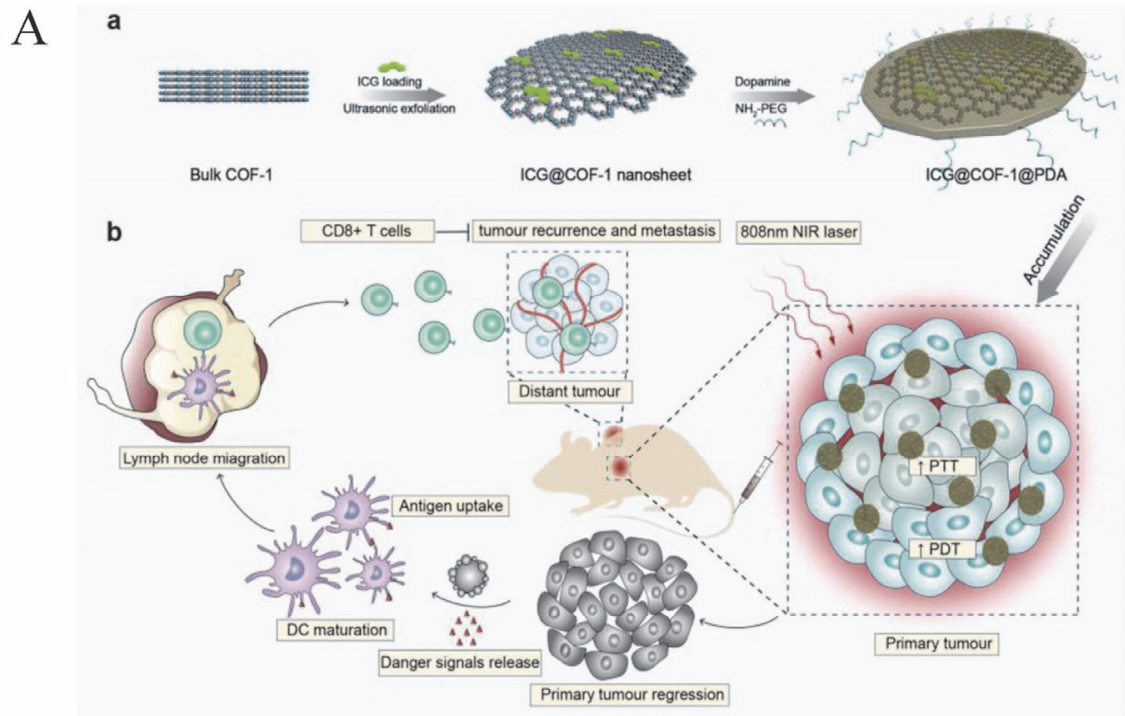

$\mathrm{B}$
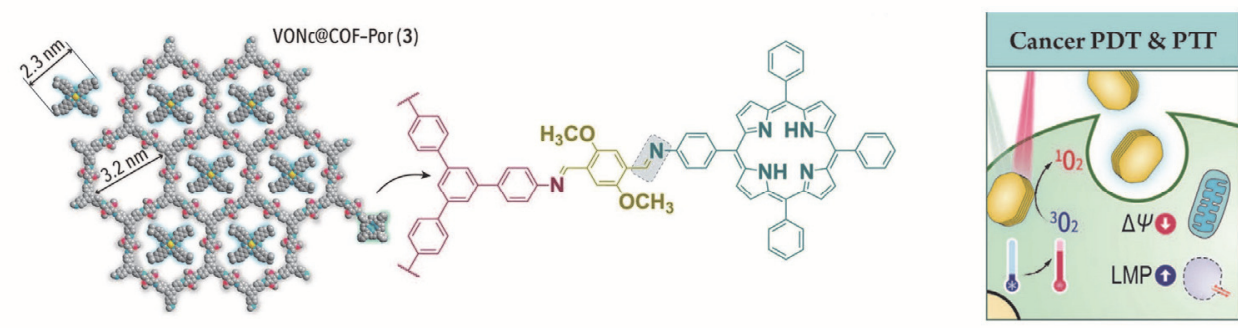

$\mathrm{C}$

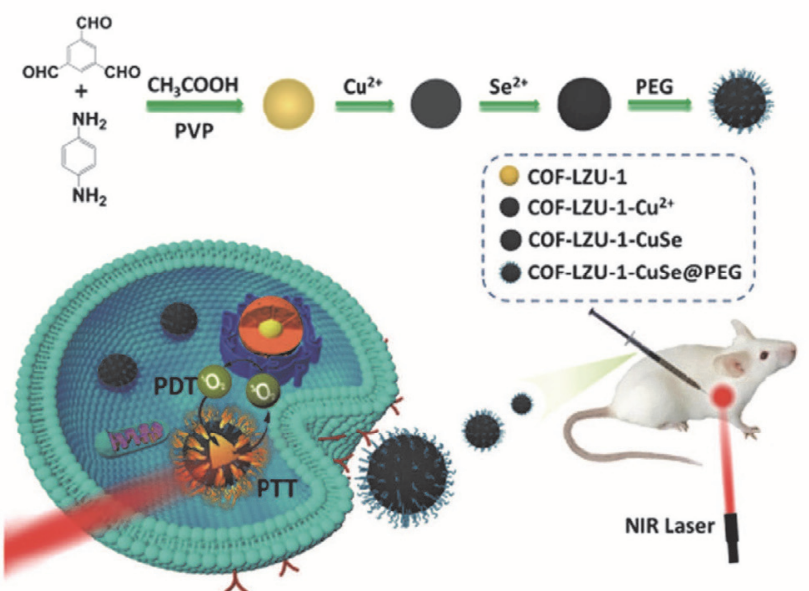

$\mathrm{D}$
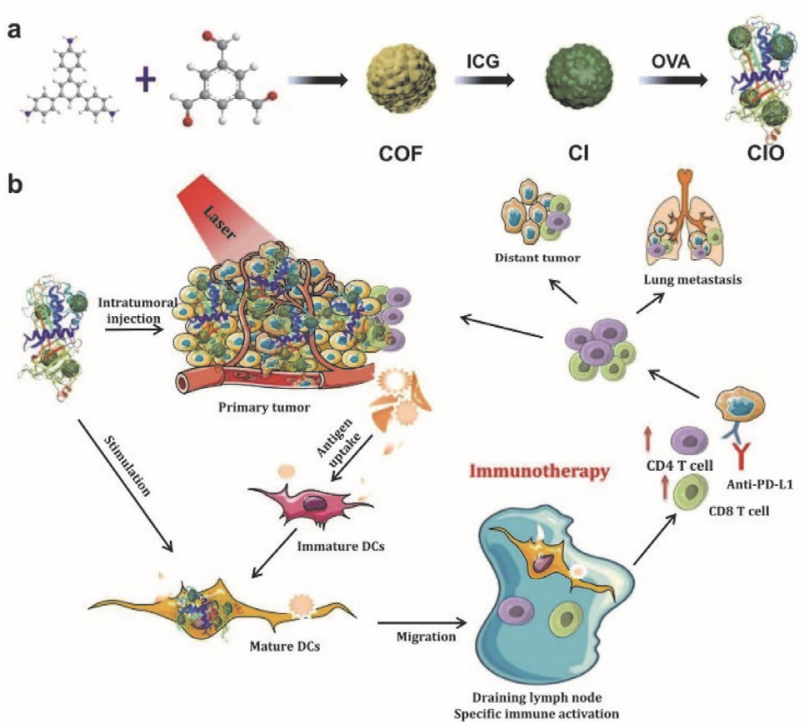

图 $8 \mathrm{COFs}$ 在光热和光动力联合治疗中的几种应用: (A) ICG@COF-1@PDA 在肿瘤治疗中的应用 ${ }^{[90]}$; (B) VONc@COF-Por 在肿瘤治疗中的应 用 ${ }^{[92]}$; (C) COF-LZU-1-CuSe @ PEG 在肿瘤治疗中的应用 ${ }^{[93]}$; (D) COF@ICG@OVA 在肿瘤治疗中的应用 ${ }^{[78]}$

Figure 8 Several applications of COFs in combined photothermal and photodynamic therapy: (A) Application of ICG@COF-1@PDA in tumor therapy $^{[90]}$; (B) application of VONc@COF-Por in tumor therapy ${ }^{[92]}$; (C) application of COF-LZU-1-CuSe@PEG in tumor therapy ${ }^{[93]}$; (D) application of COF@ICG@OVA in tumor therapy ${ }^{[78]}$

$\gamma$-SD/PLL 产生大量热量并释放出药物杀死癌细胞. 研 究表明, 经 $\gamma$-SD/PLL 和 AMF 处理 $1 \mathrm{~h}$ 后癌细胞存活率 可低至 10\%.

\section{4 结论与展望}

与其他纳米载体相比, COFs 纳米载体具有良好的 孔隙率、结晶性和生物相容性等特点, 在载药应用中表 
现出了极大的潜力. 现已将各类纳米载体优缺点进行比 较, 如表 2 所示.

在这篇综述中, 我们介绍了 COFs 的合成方法以及

A

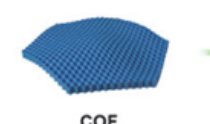

Ultrasonic exfoliation IR783

COF

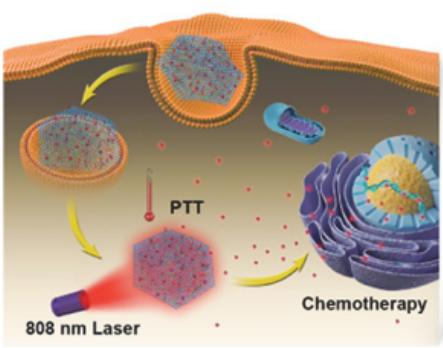

$\mathrm{C}$
在肿瘤治疗中的应用, 可以看出, 尽管 COFs 的发展目 前还处于起步阶段, 但其在药物化疗、光热疗法、光动 力疗法和联合疗法中已经取得了诸多显著成就. 然而,

A

TPB-DMTP-COF (1)
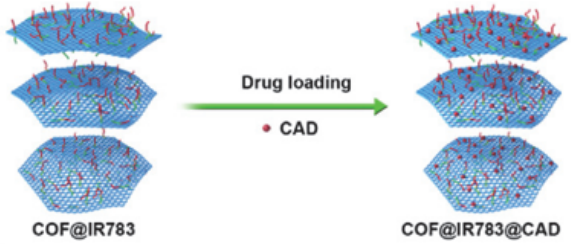

COF@IR783@CAD

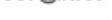

$\mathrm{B}$

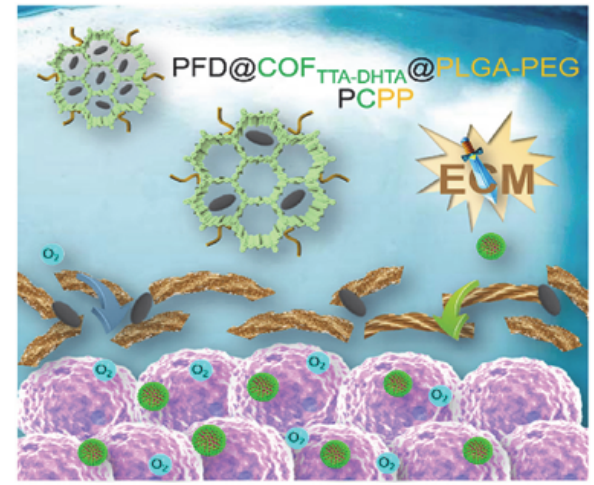

Sticar o.

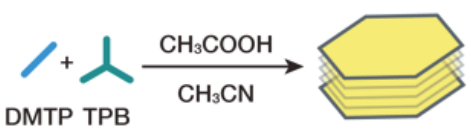

DMTP TPB

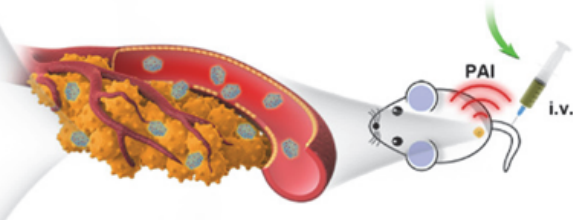

B

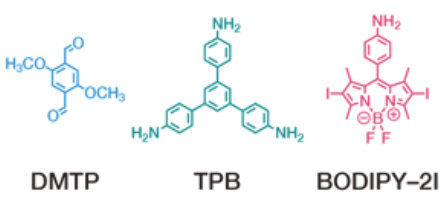

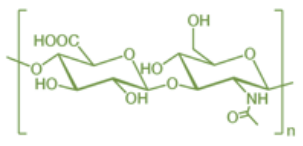

GAG
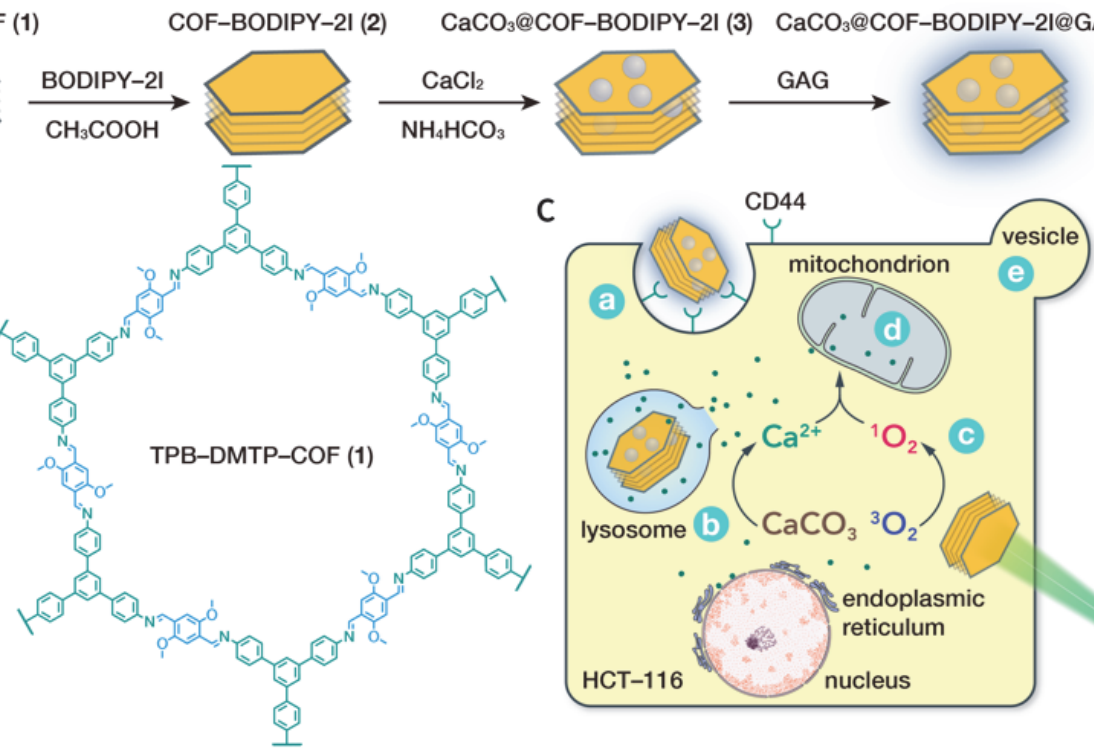

C

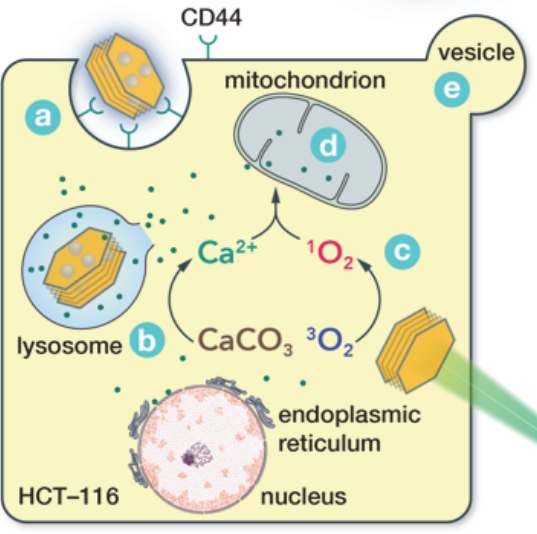

图 $9 \mathrm{COFs}$ 在光热和药物化疗以及光动力和药物化疗联合治疗中的几种应用: (A) COF@IR783@CAD 在肿瘤治疗中的应用 ${ }^{[95]}$; (B) PFD@

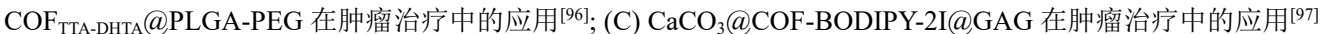

Figure 9 Several applications of COFs in photothermal and chemotherapy as well as photodynamic and chemotherapy combination therapy: (A) Appli-

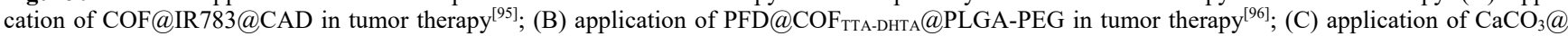
COF-BODIPY-2I@GAG in tumor therapy ${ }^{[97]}$

表 2 各类纳米载体优缺点比较

Table 2 Comparison of advantages and disadvantages of various types of nanocarriers

\begin{tabular}{|c|c|c|}
\hline 载体 & 优点 & 缺点 \\
\hline 脂质体 & $\begin{array}{l}\text { 生物相容性好、对疏水性和亲水性药物皆有较好的递送 } \\
\text { 效果 }\end{array}$ & 不易被肿瘤细胞摄取 \\
\hline 碳纳米管 & 比表面积大、易于功能化、负载效率高 & 合成成本较高、毒性较大 \\
\hline 树状大分子 & $\begin{array}{l}\text { 纳米尺寸易于控制、单分散性好、对药物分子易于控制、 } \\
\text { 生物相容性好 }\end{array}$ & 难以大量合成可用于临床纯度的产品 \\
\hline MOFs & 孔径分布均匀且可调整、比表面积大 & 易分解不稳定, 金属元素的存在使其具有细胞毒性 \\
\hline 金属氧化物 & $\begin{array}{l}\text { 稳定性好、生产成本低、形貌与粒径易于控制、拥有的 } \\
\text { 磁性使其易于监测分布情况 }\end{array}$ & 亲水性差、生物相容性较差 \\
\hline MXenes & 比表面积大、亲水性好、易功能化、稳定性好 & 合成过程污染环境、生物相容性较差 \\
\hline COFs & 孔道结构规则有序、功能多样、比表面积大、孔隙率高 & 形貌不易控制 \\
\hline
\end{tabular}


我们必须清醒地认识到, 目前的 COFs 研究还面临着以 下问题:

(1)形貌问题

虽然目前已研究出多种 COFs 的合成方法, 但仍然 没有一个理想的方案. 对于制药领域来讲, COFs 的结晶 度、孔隙度、纳米尺度粒径以及制备过程的可重复性非 常重要, 因为这些性能直接关系到 COFs 载药系统的载 药量和进入细胞的可能性. 然而现有的 COFs 合成方法 很难同时满足上述要求, 目前主要是通过自上而下或自 下而上的方法合成纳米尺度的 COFs, 然而这些方法产 率太低, 无法实现大规模生产, 这极大限制了 $\mathrm{COFs}$ 在 制药领域的应用. 上述问题仍然需要研究者们通过开发 出新的 COFs 合成方法来解决.

(2)血液中稳定性问题

$\mathrm{COFs}$ 中的可逆键导致了其对外界环境非常敏感, 稳定性较差, 然而药物载体进入生物体内后应该可以避 免被生物体清除且能够克服输送过程中的生物学障碍. 到目前为止, 很少有 COFs 可以同时满足这些要求. 目 前人们通过将惰性亲水聚合物与 COFs 结合在一定程度 缓解了这些缺陷, 然而这无法从根本上解决问题, 仍然 需要新的研究和探索.

(3)生物相容性问题

在医药领域中, 药物载体必须要安全且对生物体没 有毒副作用. COFs 作为一种新型的纳米材料, 其各项生 物相容性和毒性还未完全研究清楚, 虽然 COFs 不含有 毒的金属元素, 但是其降解产物为各种含有多个可反应 基团的多环芳香族衍生物, 这些基团是否会对生物体产 生影响仍然不得而知. 要想解决这些问题需要对其在体 内的各个过程进行仔细的研究, 而这些工作将会是一个 漫长的过程.

尽管 COFs 暂时还面临着诸多问题, 未来发展之路 任重道远, 但我们有理由相信这些问题终将会随着相关 研究的深入发展而得到解决, COFs 必将在未来肿瘤治 疗领域绽放异彩.

\section{作者简介}

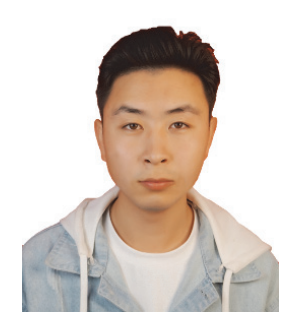

王涛, 硕士研究生. 本科毕业于山西大同大学化学与化 工学院, 2019 年起于山西大同大学化学与化工学院攻读理学 硕士学位. 主要研究方向为生物分析化学.

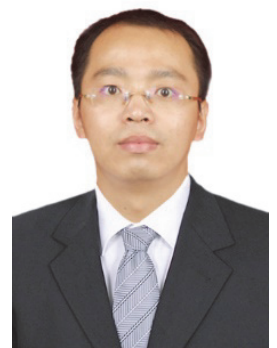

白云峰, 博士, 教授, 硕士生导师, 美国普渡大学访问学 者, 现任化学生物传感山西省重点实验室副主任. 研究方向 为生物分析化学, 近年来, 获得山西省科学技术二等奖 1 项, 山西省高等学校科学技术二等奖 1 项, 发表学术论文 60 余篇, 其中被 SCI 收录 30 余篇，已获授权国家发明专利 8 项，实用 新型专利 7 项.

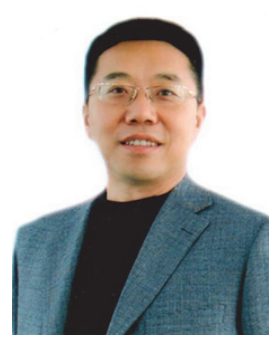

冯锋, 博士, 二级教授, 博士生导师, 享受国务院特殊津 贴专家, 现任山西大同大学校长. 新世纪百千万人才工程国 家级入选者、山西省 “三晋英才”支持计划高端领军人才、 山西省 333 人才工程入选者、山西省 131 领军人才工程学术 技术带头人、山西省学术技术带头人、山西省委联系的高级 专家、山西省优势特色学科化学学科带头人、山西省功能化 学材料重点创新团队带头人、山西省新型介孔材料的合成应 用与成果转化工程研究中心负责人、分析化学省重点建设学 科带头人、化学生物传感山西省重点实验室主任. 曾获得山西 省科学技术二等奖 2 项、山西省高等学校科学技术一等奖 2 项、山西省高等学校科学技术二等奖 1 项、山西省高等学校 优秀教学成果特等奖 1 项、山西省高等学校优秀教学成果二 等奖 2 项。已出版学术专著 2 部，在 JACS、Anal Chem、Chem Comm、Biosens Bioelectron 等杂志发表学术论文 200 余篇, 其 中被 SCI 收录论文 100 余篇, 已获授权国家发明专利 20 余项, 主持国家自然科学基金 4 项、省自然科学基金等省级科研项 目 30 余项.

\section{References}

[1] Siegel, R. L.; Miller, K. D.; Fuchs, H. E.; Jemal, A. CA Cancer J. Clin. 2021, 71, 7.

[2] Lu, H.; Goodell, V.; Disis, M. L. J. Proteome Res. 2008, 7, 1388.

[3] Xifre-Perez, E.; Guaita-Esteruelas, S.; Baranowska, M.; Pallares, J.; Masana, L.; Marsal, L. F. ACS Appl. Mater. Interfaces 2015, 7, 18600.

[4] Bai, Y.; Zhang, Z.; Cheng, L.; Wang, R.; Chen, X.; Kong, Y.; Feng, F.; Ahmad, N.; Li, L.; Liu, X. J. Biol. Chem. 2019, 294, 9911.

[5] Li, R.; Feng, F.; Chen, Z.-Z.; Bai, Y.-F.; Guo, F.-F.; Wu, F.-Y.; Zhou, G. Talanta 2015, 140, 143.

[6] Zhang, Y.; Bai, Y.; Feng, F.; Shuang, S. Anal. Methods-UK 2016, 8, 6131

[7] Bai, Y.; Zhang, H.; Zhao, L.; Wang, Y.; Chen, X.; Zhai, H.; Tian, M.; Zhao, R.; Wang, T.; Xu, H.; Feng, F. Talanta 2020, 221, 121451.

[8] Gajewski, T. F. Curr. Opin. Immunol. 2012, 6, 242.

[9] Cao, J.; Huang, D.; Peppas, N. A. Adv. Drug Deliver. Rev. 2020, 167, 
170.

[10] Fang, J.; Islam, W.; Maeda, H. Adv. Drug Deliver. Rev. 2020, 157, 142.

[11] Gu, Z.; Da Silva, C. G.; Van der Maaden, K.; Ossendorp, F.; Cruz, L. J. Pharmaceutics 2020, 12, 1054.

[12] Martincic, M.; Tobias, G. Expert Opin. Drug Deliv. 2015, 12, 563.

[13] Kesharwani, P.; Jain, K.; Jain, N. K. Prog. Polym. Sci. 2014, 39, 268.

[14] Kwon, H. J.; Shin, K.; Soh, M.; Chang, H.; Kim, J.; Lee, J.; Ko, G.; Kim, B. H.; Kim, D.; Hyeon, T. Adv. Mater. 2018, 30, e1704290.

[15] Wu, M.-X.; Yang, Y.-W. Adv. Mater. 2017, 29, 1606134.

[16] Huang, H.; Jiang, R.; Feng, Y.; Ouyang, H.; Zhou, N.; Zhang, X.; Wei, Y. Nanoscale 2020, 12, 1325.

[17] Scicluna, M. C.; Vella-Zarb, L. ACS Appl. Nano Mater. 2020, 3, 3097.

[18] Sun, T.; Lei, W.; Ma, Y.; Zhang, Y.-B. Chinese J. Chem. 2020, 38, 1153.

[19] Mei, P.; Zhang, Y.; Feng, X. Acta Chim. Sinica 2020, 78, 1041 (in Chinese). (梅佩, 张媛媛, 冯霄, 化学学报, 2020, 78, 1041.)

[20] Yu, G.; Wang, C. Chinese J. Org. Chem. 2020, 40, 1437 (in Chinese). (于歌, 汪成, 有机化学, 2020, 40, 1437.)

[21] Jiang, C.; Feng, X.; Wang, B. Acta Chim. Sinica 2020, 78, 466 (in Chinese). (蒋成浩, 冯霄, 王博, 化学学报, 2020, 78, 466.)

[22] Liu, J.; Zhang, M.; Wang, N.; Wang, C.; Ma, L. Acta Chim. Sinica 2020, 78, 311 (in Chinese). (刘建国, 张明月, 王楠, 王晨光, 马隆 龙, 化学学报, 2020, 78, 311.)

[23] Wang, W. Chinese J. Org. Chem. 2020, 40, 545 (in Chinese). (王为, 有机化学, 2020, 40, 545.)

[24] Wang, Y.; Liu, H.; Zhu, X. Acta Chim. Sinica 2020, 78, 746 (in Chinese). (王友付, 刘航海, 朱新远, 化学学报, 2020, 78, 746.)

[25] Guan, Q.; Zhou, L.-L.; Li, W.-Y.; Li, Y.-A.; Dong, Y.-B. Chem. Eur. J. 2020, 26, 5583.

[26] Feng, L.; Qian, C.; Zhao, Y. ACS Mater. Lett. 2020, 2, 1074.

[27] Bhunia, S.; Deo, K. A.; Gaharwar, A. K. Adv. Funct. Mater. 2020, 30, 2002046.

[28] Cote, A. P.; Benin, A. I.; Ockwig, N. W.; O'Keeffe, M.; Matzger, A. J.; Yaghi, O. M. Science 2005, 310, 1166.

[29] El-Kaderi, H. M.; Hunt, J. R.; Mendoza-Cortes, J. L.; Cote, A. P.; Taylor, R. E.; O'Keeffe, M.; Yaghi, O. M. Science 2007, 316, 268.

[30] Fang, Q.; Wang, J.; Gu, S.; Kaspar, R. B.; Zhuang, Z.; Zheng, J.; Guo, H.; Qiu, S.; Yan, Y. J. Am. Chem. Soc. 2015, 137, 8352.

[31] Vyas, V. S.; Vishwakarma, M.; Moudrakovski, I.; Haase, F.; Savasci, G.; Ochsenfeld, C.; Spatz, J. P.; Lotsch, B. V. Adv. Mater. 2016, 28, 8749.

[32] Geng, K.; He, T.; Liu, R.; Dalapati, S.; Tan, K. T.; Li, Z.; Tao, S.; Gong, Y.; Jiang, Q.; Jiang, D. Chem. Rev. 2020, 120, 8814.

[33] Ding, X.; Guo, J.; Feng, X.; Honsho, Y.; Guo, J.; Seki, S.; Maitarad, P.; Saeki, A.; Nagase, S.; Jiang, D. Angew. Chem. Int. Ed. 2011, 50, 1289.

[34] Nagai, A.; Chen, X.; Feng, X.; Ding, X.; Guo, Z.; Jiang, D. Angew. Chem. Int. Ed. 2013, 52, 3770.

[35] Fang, Q.; Zhuang, Z.; Gu, S.; Kaspar, R. B.; Zheng, J.; Wang, J.; Qiu, S.; Yan, Y. Nat. Commun. 2014, 5, 4503.

[36] Jiang, Y.; Huang, W.; Wang, J.; Wu, Q.; Wang, H.; Pan, L.; Liu, X. J. Mater. Chem. A 2014, $2,8201$.

[37] Thote, J.; Aiyappa, H. B.; Kumar, R. R.; Kandambeth, S.; Biswal, B. P.; Shinde, D. B.; Roy, N. C.; Banerjee, R. IUCrJ 2016, 3, 402.

[38] Wang, K.; Jia, Z.; Bai, Y.; Wang, X.; Hodgkiss, S. E.; Chen, L.; Chong, S. Y.; Wang, X.; Yang, H.; Xu, Y.; Feng, F.; Ward, J. W.; Cooper, A. I. J. Am. Chem. Soc. 2020, 142, 11131 .

[39] Zhu, L. J.; Zhang, Y. B. Molecules 2017, 22, 1149

[40] James, S. L.; Adams, C. J.; Bolm, C.; Braga, D.; Collier, P.; Friscic, T.; Grepioni, F.; Harris, K. D. M.; Hyett, G.; Jones, W.; Krebs, A.; Mack, J.; Maini, L.; Orpen, A. G.; Parkin, I. P.; Shearouse, W. C.; Steed, J. W.; Waddell, D. C. Chem. Soc. Rev. 2012, 41, 413

[41] Zhang, P.; Dai, S. J. Mater. Chem. A 2017, 5, 16118.

[42] Biswal, B. P.; Chandra, S.; Kandambeth, S.; Lukose, B.; Heine, T.; Banerjee, R. J. Am. Chem. Soc. 2013, 135, 5328.

[43] Das, G.; Shinde, D. B.; Kandambeth, S.; Biswal, B. P.; Banerjee, R. Chem. Commun. 2014, 50, 12615.

[44] Campbell, N. L.; Clowes, R.; Ritchie, L. K.; Cooper, A. I. Chem. Mater. 2009, 21, 204

[45] Ren, S. J.; Bojdys, M. J.; Dawson, R.; Laybourn, A.; Khimyak, Y. Z.; Adams, D. J.; Cooper, A. I. Adv. Mater. 2012, 24, 2357.

[46] Ritchie, L. K.; Trewin, A.; Reguera-Galan, A.; Hasell, T.; Cooper, A.
I. Micropor. Mesopor. Mat. 2010, 132, 132.

[47] Vitaku, E.; Dichtel, W. R. J. Am. Chem. Soc. 2017, 139, 12911.

[48] Kuhn, P.; Antonietti, M.; Thomas, A. Angew. Chem. Int. Ed. 2008, 47,3450

[49] Hao, Q.; Zhao, C.; Sun, B.; Lu, C.; Liu, J.; Liu, M.; Wan, L.-J.; Wang, D. J. Am. Chem. Soc. 2018, 140, 12152.

[50] Matsumoto, M.; Valentino, L.; Stiehl, G. M.; Balch, H. B.; Corcos, A. R.; Wang, F.; Ralph, D. C.; Marinas, B. J.; Dichtel, W. R. Chem 2018, 4, 308

[51] Liu, J.; Zan, W.; Li, K.; Yang, Y.; Bu, F.; Xu, Y. J. Am. Chem. Soc 2017, 139, 11666

[52] Zhou, D.; Tan, X.; Wu, H.; Tian, L.; Li, M. Angew. Chem. Int. Ed. 2019, 58,1376

[53] Ding, S.-Y.; Wang, W. Chem. Soc. Rev. 2013, 42, 548.

[54] Ding, S.-Y.; Cui, X.-H.; Feng, J.; Lu, G.; Wang, W. Chem. Commun. 2017, 53, 11956.

[55] Medina, D. D.; Rotter, J. M.; Hu, Y.; Dogru, M.; Werner, V.; Auras, F.; Markiewicz, J. T.; Knochel, P.; Bein, T. J. Am. Chem. Soc. 2015 137, 1016.

[56] Guan, Q.; Wang, G.-B.; Zhou, L.-L.; Li, W.-Y.; Dong, Y.-B. Nanoscale Adv. 2020, 2, 3656.

[57] Berlanga, I.; Ruiz-González, M. L.; González-Calbet, J. M.; Fierro, J. L. G.; Mas-Ballesté, R.; Zamora, F. Small 2011, 7, 1207.

[58] Chandra, S.; Kandambeth, S.; Biswal, B. P.; Lukose, B.; Kunjir, S. M.; Chaudhary, M.; Babarao, R.; Heine, T.; Banerjee, R. J. Am. Chem. Soc. 2013, 135, 17853.

[59] Wang, K.; Zhang, Z.; Lin, L.; Chen, J.; Hao, K.; Tian, H.; Chen, X. Chem. Mater. 2019, 31, 3313

[60] Kong, W.; Wan, J.; Namuangruk, S.; Guo, J.; Wang, C. Sci. Rep. 2018, 8, 5529 .

[61] Dong, J.; Li, X.; Peh, S. B.; Yuan, Y. D.; Wang, Y.; Ji, D.; Peng, S.; Liu, G.; Ying, S.; Yuan, D.; Jiang, J.; Ramakrishna, S.; Zhao, D. Chem. Mater. 2019, 31, 146 .

[62] Smith, B. J.; Parent, L. R.; Overholts, A. C.; Beaucage, P. A.; Bisbey, R. P.; Chavez, A. D.; Hwang, N.; Park, C.; Evans, A. M.; Gianneschi, N. C.; Dichtel, W. R. ACS Central Sci. 2017, 3, 58.

[63] Das, G.; Prakasam, T.; Addicoat, M. A.; Sharma, S. K.; Ravaux, F.; Mathew, R.; Baias, M.; Jagannathan, R.; Olson, M. A.; Trabolsi, A. J. Am. Chem. Soc. 2019, 141, 19078.

[64] Matsumoto, M.; Dasari, R. R.; Ji, W.; Feriante, C. H.; Parker, T. C.; Marder, S. R.; Dichtel, W. R. J. Am. Chem. Soc. 2017, 139, 4999.

[65] Li, M.; Qiao, S.; Zheng, Y.; Andaloussi, Y. H.; Li, X.; Zhang, Z.; Li, A.; Cheng, P.; Ma, S.; Chen, Y. J. Am. Chem. Soc. 2020, 142, 6675.

[66] Tan, J.; Namuangruk, S.; Kong, W. F.; Kungwan, N.; Guo, J.; Wang, C. C. Angew. Chem. Int. Ed. 2016, 55, 13979.

[67] Bai, L.; Phua, S. Z.; Lim, W. Q.; Jana, A.; Luo, Z.; Tham, H. P.; Zhao, L.; Gao, Q.; Zhao, Y. Chem. Commun. 2016, 52, 4128

[68] Mitra, S.; Sasmal, H. S.; Kundu, T.; Kandambeth, S.; Illath, K.; Diaz Diaz, D.; Banerjee, R. J. Am. Chem. Soc. 2017, 139, 4513.

[69] Zhang, G.; Li, X.; Liao, Q.; Liu, Y.; Xi, K.; Huang, W.; Jia, X. Nat. Commun. 2018, 9, 2785.

[70] Liu, S.; Hu, C.; Liu, Y.; Zhao, X.; Pang, M.; Lin, J. Chem. Eur. J. 2019, 25, 4315

[71] Lal, S.; Clare, S. E.; Halas, N. J. Acc. Chem. Res. 2008, 41, 1842.

[72] Gallego, N. C.; Klett, J. W. Carbon 2003, 41, 1461.

[73] Mi, Z.; Yang, P.; Wang, R.; Unruangsri, J.; Yang, W.; Wang, C.; Guo, J. J. Am. Chem. Soc. 2019, 141, 14433.

[74] Lan, M.; Zhao, S.; Liu, W.; Lee, C.-S.; Zhang, W.; Wang, P. Adv. Healthc. Mater. 2019, 8, 1900132 .

[75] Kharkwal, G. B.; Sharma, S. K.; Huang, Y.-Y.; Dai, T.; Hamblin, M. R. Lasers Surg. Med. 2011, 43, 755.

[76] Thakor, A. S.; Gambhir, S. S. CA-Cancer J. Clin. 2013, 63, 395.

[77] Bhanja, P.; Mishra, S.; Manna, K.; Mallick, A.; Das Saha, K.; Bhaumik, A. ACS Appl. Mater. Interfaces 2017, 9, 31411.

[78] Zhou, Y.; Liu, S. N.; Hu, C. L.; Cai, L. H.; Pang, M. L. J. Mater. Chem. B 2020, 8, 5451 .

[79] Guan, Q.; Fu, D.-D.; Li, Y.-A.; Kong, X.-M.; Wei, Z.-Y.; Li, W.-Y.; Zhang, S.-J.; Dong, Y.-B. iScience 2019, 14, 180.

[80] Zhang, Y.; Zhang, L.; Wang, Z.; Wang, F.; Kang, L.; Cao, F.; Dong, K.; Ren, J.; Qu, X. Biomaterials 2019, 223, 119462.

[81] Zhang, L.; Wang, S. B.; Zhou, Y.; Wang, C.; Zhang, X. Z.; Deng, H. X. Angew. Chem. Int. Ed. 2019, 58, 14213.

[82] Cai, L.; Hu, C.; Liu, S.; Zhou, Y.; Pang, M.; Lin, J. Sci. China Mater. 2021, 64, 488.

[83] Wang, P.; Zhou, Z.; Guan, K. S.; Wang, Y. J.; Fu, X. Y.; Yang, Y.; 
Yin, X.; Song, G. S.; Zhang, X. B.; Tan, W. H. Chem. Sci. 2020, 11, 1299.

[84] Chen, Z. K.; Liu, L. L.; Liang, R. J.; Luo, Z. Y.; He, H. M.; Wu, Z. H.; Tian, H.; Zheng, M. B.; Ma, Y. F.; Cai, L. T. ACS Nano 2018, 12 , 8633.

[85] Li, X. S.; Kwon, N.; Guo, T.; Liu, Z.; Yoon, J. Angew. Chem. Int. Ed. 2018, 57, 11522 .

[86] Reisch, A.; Klymchenko, A. S. Small 2016, 12, 1968.

[87] Yang, G.; Xu, L.; Chao, Y.; Xu, J.; Sun, X.; Wu, Y.; Peng, R.; Liu, Z. Nat. Commun. 2017, 8, 902.

[88] Zhang, H.; Li, G.; Liao, C.; Cai, Y.; Jiang, G. J. Mater. Chem. B 2019, 7, 2398

[89] Feng, J.; Ren, W.-X.; Kong, F.; Dong, Y.-B. Inorg. Chem. Front. 2021, 8, 848.

[90] Gan, S.; Tong, X.; Zhang, Y.; Wu, J.; Hu, Y.; Yuan, A. Adv. Funct. Mater. 2019, 29, 1902757.

[91] Wang, D.; Zhang, Z.; Lin, L.; Liu, F.; Wang, Y.; Guo, Z.; Li, Y.; Tian, H.; Chen, X. Biomaterials 2019, 223, 119459.
[92] Guan, Q.; Zhou, L.-L.; Li, Y.-A.; Li, W.-Y.; Wang, S.; Song, C.; Dong, Y.-B. ACS Nano 2019, 13, 13304

[93] Hu, C.; Zhang, Z.; Liu, S.; Liu, X.; Pang, M. ACS Appl. Mater. Interfaces 2019, 11, 23072

[94] Liu, S.; Zhou, Y.; Hu, C.; Cai, L.; Pang, M. ACS Appl. Mater. Interfaces 2020, 12, 43456.

[95] Wang, K.; Zhang, Z.; Lin, L.; Hao, K.; Chen, J.; Tian, H.; Chen, X. ACS Appl. Mater. Interfaces 2019, 11, 39503.

[96] Wang, S.-B.; Chen, Z.-X.; Gao, F.; Zhang, C.; Zou, M.-Z.; Ye, J.-J.; Zeng, X.; Zhang, X.-Z. Biomaterials 2020, 234, 119772.

[97] Guan, Q.; Zhou, L.-L.; Lv, F.-H.; Li, W.-Y.; Li, Y.-A.; Dong, Y.-B. Angew. Chem. Int. Ed. 2020, 59, 18042.

[98] Benyettou, F.; Das, G.; Nair, A. R.; Prakasam, T.; Shinde, D. B.; Sharma, S. K.; Whelan, J.; Lalatonne, Y.; Traboulsi, H.; Pasricha, R.; Abdullah, O.; Jagannathan, R.; Lai, Z.; Motte, L.; Gándara, F.; Sadler, K. C.; Trabolsi, A. J. Am. Chem. Soc. 2020, 142, 18782.

(Cheng, B.) 